\title{
Three classes of epigenomic regulators converge to hyperactivate the essential maternal gene deadhead within a heterochromatin mini-domain
}

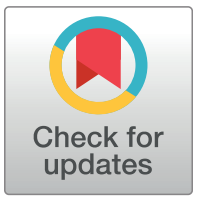

\section{G OPEnACCESS}

Citation: Torres-Campana D, Horard B, Denaud S, Benoit G, Loppin B, Orsi GA (2022) Three classes of epigenomic regulators converge to hyperactivate the essential maternal gene deadhead within a heterochromatin mini-domain. PLoS Genet 18(1): e1009615. https://doi.org/10.1371/journal. pgen.1009615

Editor: Giovanni Bosco, Geisel School of Medicine at Dartmouth, UNITED STATES

Received: May 18, 2021

Accepted: December 10, 2021

Published: January 4, 2022

Copyright: ๑ 2022 Torres-Campana et al. This is an open access article distributed under the terms of the Creative Commons Attribution License, which permits unrestricted use, distribution, and reproduction in any medium, provided the original author and source are credited.

Data Availability Statement: All original sequencing data files from this study are publicly available from the GEO repository under the accession numbers GSE174263 and GSE174250. Additional sequencing data used in this study are available from GEO under the following accession numbers: GSE151981 (ATAC-seq), GSE37444 and GSE145282 (H3K27me3 ChIP-seq), GSE99027 (H3K9me3 ChIP-seq), GSE36393 (Mod(mdg4) ChIP-seq), GSE62904 (CP190, Beaf-32 and Dref ChIP-seq) and GSE24521 (Polycomb and

\author{
Daniela Torres-Campana $\oplus^{1}$, Béatrice Horard ${ }^{1}$, Sandrine Denaud $\varpi^{2}$, Gérard Benoit $\oplus^{1}$, \\ Benjamin Loppin $\odot^{1 *}$, Guillermo A. Orsi $\odot^{1{ }^{1 a}}{ }^{1}$ \\ 1 Laboratoire de Biologie et Modélisation de la Cellule, CNRS UMR5239, École Normale Supérieure de \\ Lyon, University of Lyon, Lyon, France, 2 Institute of Human Genetics, UMR 9002, CNRS, Université de \\ Montpellier, Montpellier, France \\ a Current address: Institute for Advanced Biosciences, INSERM U1209, CNRS, Université Grenoble-Alpes, \\ Grenoble, France \\ * benjamin.loppin@ens-lyon.fr (BL); guillermo.orsi@inserm.fr (GAO)
}

\section{Abstract}

The formation of a diploid zygote is a highly complex cellular process that is entirely controlled by maternal gene products stored in the egg cytoplasm. This highly specialized transcriptional program is tightly controlled at the chromatin level in the female germline. As an extreme case in point, the massive and specific ovarian expression of the essential thioredoxin Deadhead (DHD) is critically regulated in Drosophila by the histone demethylase Lid and its partner, the histone deacetylase complex $\operatorname{Sin} 3 \mathrm{~A} / \mathrm{Rpd} 3$, via yet unknown mechanisms. Here, we identified Snr1 and Mod(mdg4) as essential for dhd expression and investigated how these epigenomic effectors act with Lid and Sin3A to hyperactivate $d h d$. Using Cut\&Run chromatin profiling with a dedicated data analysis procedure, we found that $d h d$ is intriguingly embedded in an H3K27me3/H3K9me3-enriched mini-domain flanked by DNA regulatory elements, including a dhd promoter-proximal element essential for its expression. Surprisingly, Lid, Sin3a, Snr1 and $\mathrm{Mod}(\mathrm{mdg} 4)$ impact H3K27me3 and this regulatory element in distinct manners. However, we show that these effectors activate $d h d$ independently of $\mathrm{H} 3 \mathrm{~K} 27 \mathrm{me} / \mathrm{H} 3 \mathrm{~K} 9 \mathrm{me} 3$, and that $d h d$ remains silent in the absence of these marks. Together, our study demonstrates an atypical and critical role for chromatin regulators Lid, Sin3A, Snr1 and Mod(mdg4) to trigger tissue-specific hyperactivation within a unique heterochromatin mini-domain.

\section{Author summary}

Multicellular development depends on a tight control of gene expression in each cell type. This relies on the coordinated activities of nuclear proteins that interact with DNA or its histone scaffold to promote or restrict gene transcription. For example, we previously showed that the histone modifying enzymes Lid and Sin3A/Rpd3 are required in Drosophila ovaries for the massive expression of deadhead (dhd), a gene encoding for a thioredoxin that is 
Polyohomeotic ChIP-seq). All other relevant data are within the manuscript and its supporting information files.

Funding: This work was supported by an Association pour la Recherche sur le Cancer (ARC) Foundation grant (PJA20191209671) to BL. DTC was supported by a PhD fellowship from the Ministère de l'Enseignement supérieur, de la Recherche et de l'Innovation and from the ARC (ARCDOC42020020001727). The funders had no role in study design, data collection and analysis, decision to publish or preparation of the manuscript.

Competing interests: The authors have declared that no competing interests exist. essential for fertility. In this paper, we have further identified two additional $d h d$ regulators, Snrl and Mod(mdg4) and dissected the mechanism behind hyperactivation of this gene. Using the epigenomic profiling method Cut\&Run with a dedicated data analysis approach, we unexpectedly found that $d h d$ is embedded in an unusual chromatin mini-domain featuring repressive histone modifications $\mathrm{H} 3 \mathrm{~K} 27 \mathrm{me} 3$ and $\mathrm{H} 3 \mathrm{~K} 9 \mathrm{me} 3$ and flanked by two regulatory elements. However, we further showed that Lid, Sin3A, Snr1 and Mod(mdg4) behave like obligatory activators of $d h d$ independently of this mini-domain. Our study unveils how multiple broad-acting epigenomic effectors operate in non-canonical manners to ensure a critical and specialized gene activation event. These findings challenge our knowledge on these regulatory mechanisms and their roles in development and pathology.

\section{Introduction}

Gene expression is tightly controlled in eukaryotic cells by the composition, organization and dynamics of nucleosomes, consisting of an octamer of histone proteins wrapped in $\sim 146 \mathrm{bp}$ of DNA. The concerted activity of protein complexes including histone chaperones, readers and writers as well as nucleosome remodelers, defines the positioning, composition and post-translational modifications of nucleosomes [1-3]. The resulting chromatin landscape is further organized by insulator proteins that delimit tridimensional contacts along the genome, forming sub-nuclear domains and guiding contacts between promoters and their cognate regulatory elements [4]. This tightly regulated epigenomic environment profoundly influences RNA Polymerase access to DNA and transcriptional activity.

Tremendous efforts in the past decades aimed at dissecting the roles of these epigenomic effectors in vivo. A privileged method is ablation or dosage manipulation of each component to measure its impact on gene expression. While these approaches can yield precious functional insight, the ubiquitous expression and wide range of activities of these factors, as well as redundancies in their interactions, make it difficult to infer their precise function. Understanding their function therefore requires identifying biologically relevant situations where disrupting these effectors impacts transcription in a critical and specific manner. We previously described one of such cases, where perturbation of the histone H3K4 demethylase Lid/KDM5 or the histone deacetylase complex Sin3A/Rpd3 in Drosophila ovaries dramatically abrogated the expression of the maternal gene deadhead ( $d h d$ ), which is essential for female fertility [5].

The Drosophila egg is loaded with maternal gene products synthesized by germline nurse cells that enable early embryonic development in the absence of zygotic transcription [6]. An extreme example of this specialized transcriptome, $d h d$ is among the most highly expressed genes in adult ovaries, while it is almost completely silent in any other tissue and developmental stage [5,7-9]. The DHD protein is a thioredoxin involved in regulating the general redox state in oocytes $[10,11]$. In addition, DHD plays a critical role at fertilization to reduce cysteine-cysteine disulfide bonds on the Protamine-like proteins that replace histones on chromatin during spermiogenesis $[9,12]$. In the absence of DHD, paternal chromosomes fail to decondense and are excluded from the first zygotic nucleus, leading to haploid gynogenetic development and embryonic lethality. The $d h d$ locus, which produces a single, short (952bp), intronless transcript is packed within a $1369 \mathrm{bp}$ region that separates its flanking genes $\operatorname{Tr} x-T$ and CG4198. Remarkably, these two genes are expressed exclusively in the male germline, thereby constituting an apparently unfavorable environment for $d h d$ transcription in ovaries. In addition, we showed that a 4305bp transgene spanning only Trx-T, dhd and part of CG4198 largely recapitulates the expression of $d h d[5,9]$, indicating that regulatory elements sufficient 
for $d h d$ activation are contained within this restricted region. Our previous study further found that Lid and Sin3A are essential activators of $d$ hd in Drosophila ovaries, in striking contrast to their otherwise relatively modest impact on the rest of the transcriptome. Considering these unusual features, we postulated that the exquisite sensitivity of $d h d$ to these broad-acting chromatin effectors revealed a singular mode of epigenomic regulation that enables its massive and specific ovarian expression [5].

Here, we exploited this singular model locus to understand how multiple classes of epigenomic effectors converge to achieve programmed transcriptional hyperactivation. We identified the Brahma chromatin remodeler component Snr1 [13] and the BTB/POZ-domain protein Mod(mdg4) [14] as factors that share with Lid and Sin3A a critical and highly specific role in activating dhd. By exploiting the chromatin profiling method Cut\&Run [15] and an adapted data analysis strategy, we found that $d h d$ is unexpectedly embedded within a heterochromatin mini-domain flanked by two border regulatory elements. One of these is a dhdproximal element, which encompasses a DNA Replication-related Element (DRE-box) motif [16] that is essential for $d h d$ expression. Yet, exploiting knockdown and transgenic tools, we found that Lid, Sin3A, Snr1 and Mod(mdg4) activate $d h d$ independently of the associated heterochromatin mini-domain. Furthermore, this mini-domain is not required to restrict $d h d$ expression to ovaries. Together, our results put into perspective our understanding on these epigenomic regulators by revealing how they exert a biologically essential control of $d h d$ via non-canonical mechanisms.

\section{Results}

\section{$\operatorname{Mod}(\operatorname{mdg} 4)$ and Snr1 are essential for $d h d$ expression}

We previously performed a female germline RNA interference screen to identify chromatin factors required for paternal chromosome incorporation into the zygote at fertilization [5]. As part of that screen, the histone H3K4 demethylase Lid, Sin3A and Rpd3, which participate in deacetylase complexes targeting various lysine residues in $\mathrm{H} 3$ and $\mathrm{H} 4[17,18]$, were identified as essential regulators of $d h d$ expression. Because Lid and Sin3A can interact within a corepressor complex $[19,20]$, we asked whether other chromatin regulatory complexes might also be involved in $d h d$ regulation. We therefore broadened our analysis to other knockdowns that caused maternal effect sterility associated with a $d h d$-like mutant phenotype, i.e. defective sperm nuclear decompaction at fertilization. Among these, we focused on two additional UAS-controlled small hairpin RNA (shRNA) constructs from the TRiP collection [21], respectively targeting $\bmod (\operatorname{mdg} 4)$ and Snr1. Snrl is an essential subunit of the Brahma chromatin remodeler that mediates protein-protein interactions within this complex as well as with external interacting partners [13,22]. The $\bmod (m d g 4)$ gene codes for up to 31 isoforms [23], all of which are targeted by the shRNA construct. Among these, the most well characterized, Mod (mdg4)67.2 is a common component of boundary insulators in the Drosophila genome [24], but other non-insulating isoforms exhibiting activator functions have also been identified $[4,25,26]$. These two candidates belonged to two classes of epigenomic effectors distinct from Lid and Sin $3 \mathrm{~A}$, and we thus decided to investigate their function during the oocyte to zygote transition.

When activated by the Maternal Triple Driver (MTD) Gal4 source, these shRNAs efficiently reduced the levels of $\bmod (m d g 4)$ and $S n r 1$ transcripts (S1A Fig). Previous studies reported defective oogenesis and diminished egg production in $\bmod (\operatorname{mdg} 4)$ as well as Snrl mutant females [22,25]. Interestingly, females with ovarian knockdown of mod(mdg4) or Snrl (hereby referred to as $\bmod (\operatorname{mdg} 4) \mathrm{KD}$ or Snr1 KD females) were almost completely sterile (Table 1). Indeed, while $\mathrm{KD}$ females were able to lay more eggs than mutants, these almost systematically 
Table 1. Embryo hatching rates. The $w^{1118}$ strain is used as reference.

\begin{tabular}{|c|c|c|c|}
\hline \multicolumn{4}{|c|}{ Knockdowns } \\
\hline Female Genotype & Male Genotype & Number of eggs & Hatch. rate \\
\hline Control & $w^{1118}$ & 1561 & $98.27 \%$ \\
\hline Snr1 KD & $w^{1118}$ & 791 & $0.00 \%$ \\
\hline $\bmod (m d g 4) \mathrm{KD}$ & $w^{1118}$ & 1589 & $1.01 \%$ \\
\hline lid KD (val22) & $w^{1118}$ & 1403 & $2.14 \%$ \\
\hline lid $\mathrm{KD}$ (val21) & $w^{1118}$ & 1144 & $1.05 \%$ \\
\hline $\operatorname{Sin} 3 a \mathrm{KD}$ & $w^{1118}$ & 1221 & $0.25 \%$ \\
\hline$E(z) \mathrm{KD}$ & $w^{1118}$ & 843 & $0.00 \%$ \\
\hline \multicolumn{4}{|c|}{ Rescue with WT or $\triangle D R E$ mutant transgene } \\
\hline$w^{1118}$ & $w^{1118}$ & 344 & $97.67 \%$ \\
\hline$d h d^{J 5}$ & $w^{1118}$ & 375 & $0.00 \%$ \\
\hline$d h d^{J 5 ;} ; p W 8-d h d^{W T}$ & $w^{1118}$ & 663 & $85.67 \%$ \\
\hline$d h d^{J 5} ; ; p W 8-d h d^{\Delta D R E}$ & $w^{1118}$ & 475 & $2.15 \%$ \\
\hline$d h d^{J 5} ; p W 8-d h d^{F D}$ & $w^{1118}$ & 410 & $87.80 \%$ \\
\hline \multicolumn{4}{|c|}{ Knockdown rescue with the WT transgene } \\
\hline 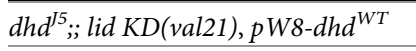 & $w^{1118}$ & 175 & $1.92 \%$ \\
\hline$d h d^{55} ; ; \operatorname{Sin} 3 a K D, p W 8-d h d^{W T}$ & $w^{1118}$ & 271 & $0.37 \%$ \\
\hline$d h d^{5} ; ; \operatorname{Snr} 1 \mathrm{KD}, p W 8-d h d^{W T}$ & $w^{1118}$ & 247 & $0.00 \%$ \\
\hline$d h d^{J 5} ; \bmod (m d g 4) K D, p W 8-d h d^{W T}$ & $w^{1118}$ & 462 & $0.43 \%$ \\
\hline
\end{tabular}

https://doi.org/10.1371/journal.pgen.1009615.t001

failed to hatch. Focusing on paternal chromatin organization at fertilization in these embryos, we found that both $\bmod (m d g 4)$ and Snrl ovarian KDs systematically led to failure of male pronucleus decondensation, which remained elongated (Fig 1A). Concomitantly, these eggs exhibited retention of the protamine fluorescent marker Mst35Ba::GFP (ProtA::GFP) [27] in paternal chromatin, as observed in $d h d$ loss of function mutants $[9,12]$.

The above results suggest that $\operatorname{Mod}(\operatorname{mg} 4)$ and Snr1 could regulate $d h d$ expression. RNAsequencing on $\bmod (\operatorname{mdg} 4)$ and $S n r 1 \mathrm{KD}$ ovaries indeed revealed that $d h d$ is dramatically downregulated in both KDs, with a fold reduction of almost two orders of magnitude (Figs 1B, $1 \mathrm{C}, \mathrm{S} 1 \mathrm{~B}$ and S1E). dhd was the first most strongly affected gene in mod(mdg4) KD ovaries in terms of fold-change in expression, and the $14^{\text {th }}$ most affected gene in Snr1 KD ovaries. Consistently, DHD protein levels assessed by Western Blot in KD ovaries were also dramatically reduced (Fig 1D). This was in contrast to a more modest impact of both KDs on the rest of the transcriptome and the limited overlap in their effects (S1B, S1C and S1D Fig). In particular, genes in the vicinity of $d h d$ were not significantly affected by the KDs (Figs 1C and S1B). Therefore, despite the packed genomic organization of the $d h d$ locus, its expression strictly and singularly depends on multiple epigenomic effectors.

\section{Cut\&Run with dedicated analysis reveals both the distribution of histone modifications and their associated regulatory elements}

To more precisely characterize the chromatin landscape at the $d h d$ locus, we next implemented the Cut\&Run epigenomic profiling method [15]. In Cut\&Run, histone modifications of interest are targeted in situ by a specific antibody following tissue permeabilization. Target-bound antibodies are subsequently coupled to a fusion between the bacterial Protein A and Micrococcal Nuclease (ProteinA-MNase) that cleaves exposed DNA in the vicinity of the antibody, releasing target nucleosomal particles into solution. Importantly, MNase is expected to also cleave exposed DNA in the immediate spatial vicinity of the nucleosome-bound antibody, causing 


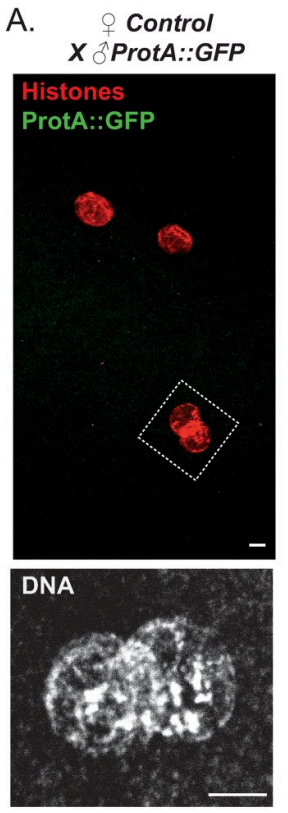

B

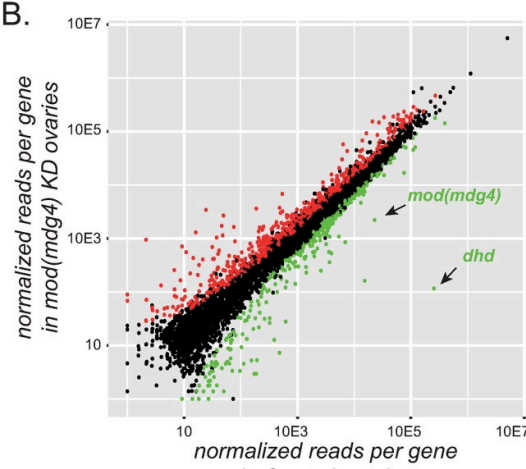

in Control ovaries

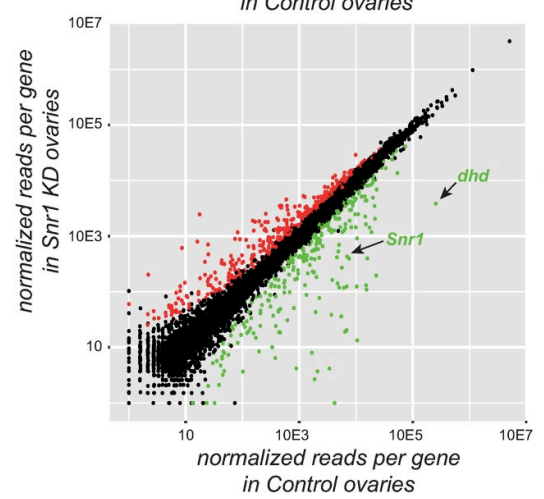

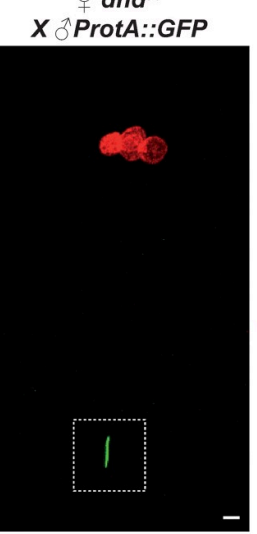
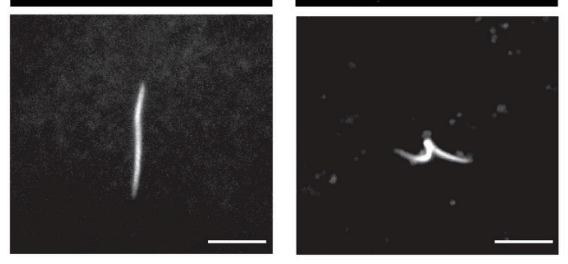

$\uparrow \bmod (\operatorname{mdg} 4) K D$

$X$ ProtA::GFP
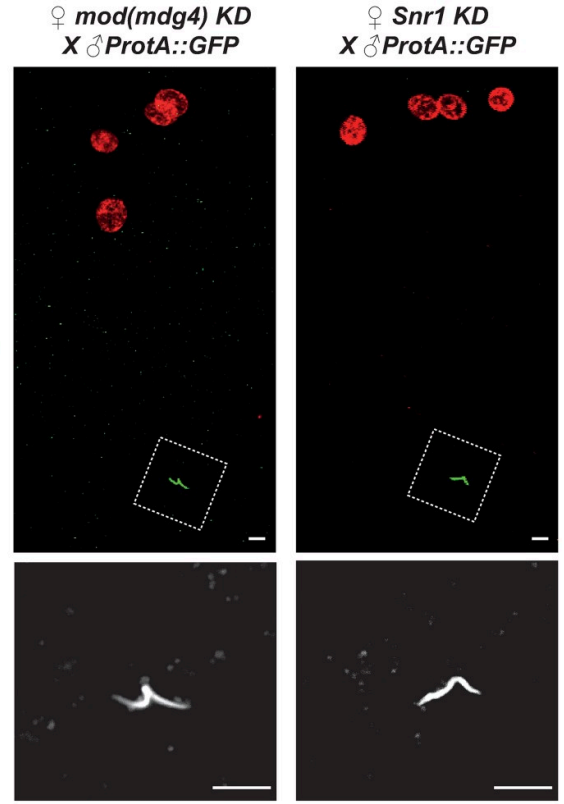

C. $\quad(10-170001$

$1 \mathrm{~kb}$
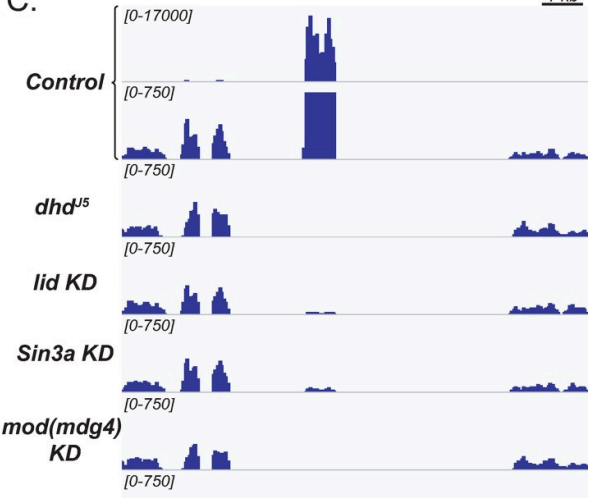

Snr1 KD

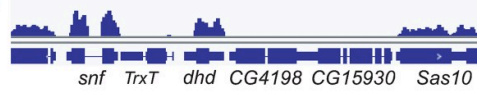

D.

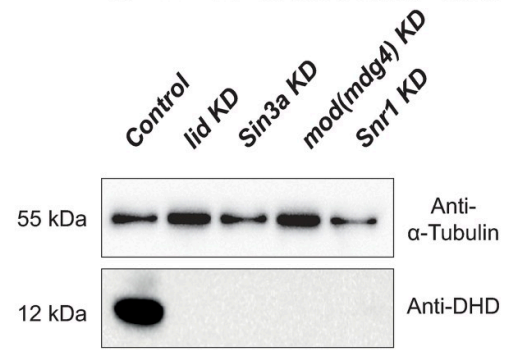

Fig 1. Mod(mdg4) and Snr1 are required for $d h d$ expression. A-Maternal Mod(mdg4) and Snr1 are required for protamine removal and sperm nuclear decompaction at fertilization. Top: Confocal images of pronuclear apposition in eggs from Control $(M T D>+)$, dhd ${ }^{55}$, mod(mdg4) KD or Snrl KD females mated with transgenic ProtA::GFP males. The sperm nucleus in $d h d^{5}$, $\bmod (m d g 4) \mathrm{KD}$ and Snrl KD eggs retains ProtA::GFP (green) and has a needle-shape morphology. Bottom: zoom on the sperm nucleus. Scale bars: $5 \mu \mathrm{m}$. B-dhd is strongly downregulated in $\bmod (\operatorname{mdg} 4)$ $\mathrm{KD}$ and Snr1 KD ovaries. RNA-seq normalized reads per gene (in RPKM) are shown for $\bmod (\operatorname{mdg} 4) \mathrm{KD}$ vs Control (top) and Snrl KD vs Control (bottom). Genes downregulated (green) or upregulated (red) in KD ovaries are highlighted. C-Genome Browser view of Control, $d h d^{5}$, lid KD, Sin3a KD, mod(mdg4) KD and Snrl KD ovarian RNA-seq signal at the $d h d$ region showing dramatic downregulation in all KD conditions. Note that the Control track is represented at two different scales to fit the high read count for $d h d$ (top track) or the low read count for its neighboring genes (bottom track). D-The DHD protein is undetectable in KD ovaries. Western blot analysis using an anti-DHD antibody on ovary extracts of the indicated genotypes. Alpha-tubulin is used as a loading control.

https://doi.org/10.1371/journal.pgen.1009615.g001 
the release of DNA particles bound by other proteins such as polymerases or DNA sequence-specific transcription factors (Fig 2A). In particular, DNA regulatory elements occupied by sequencespecific transcription factors are typically associated with MNase footprints distinctly shorter than nucleosomes [28-30]. Partially unwrapped dynamic nucleosomes typically associated with regulatory elements can also produce such distinctly short footprints [31]. Following paired-end sequencing, such released DNA fragments can be distinguished and separated by their size, yielding a map of nucleosomes $(>146 \mathrm{bp}$ ) and sub-nucleosomal particles, putatively corresponding to regulatory elements $(<120 \mathrm{bp})$. A single Cut\&Run experiment should thus identify DNA regulatory elements that are in physical proximity of target histone modifications.

With this in mind, we conducted H3K27me3 Cut\&Run in Drosophila ovaries. Using only 12 pairs of ovaries per sample, we robustly revealed H3K27me3 domains. Remarkably, visualization of Cut\&Run fragments shorter than $120 \mathrm{bp}$ (which excludes fully wrapped octameric nucleosomes) revealed that these were enriched at discrete peaks within H3K27me3 domains. Genome-wide analysis identified 679 peaks of fragments $<120 \mathrm{bp}$ (hereon referred to as "short fragment peaks") (Fig 2B and 2D). We hypothesized that short fragment peaks represented H3K27me3-associated regulatory elements occupied by transcription factors. Within H3K27me3 domains, we expected these to include Polycomb Response Elements (PREs) as well as insulators. For example, short fragment peaks corresponded to several well-described PREs and insulators in the Bithorax complex H3K27me3 domain [26,32,33] (Fig 2C), consistent with observations in larval tissue [34]. To ask whether this reflects a broader genome-wide trend, we compared short fragment peaks with PRE and insulator markers genome-wide. Although there is scarce genome-wide data available for Drosophila ovaries, H3K27me3 domains are generally present in most cell types. We thus exploited datasets from embryonicderived S2 and Kc cell lines. Consistent with their occupancy by transcription factors, ATACseq peaks [35] -revealing hyper-accessible DNA- coincide with Polycomb regulatory elements in flies and mice $[35,36]$. Genome-wide, our small fragment peaks identified in ovaries were enriched for ATAC-seq signal, arguing that these indeed correspond to DNA regulatory elements (Fig 2D). Enrichment at these peaks of the Polycomb protein [37] and the insulator protein CP190 [38] further argues that these elements often correspond to functional PREs or insulators. Accordingly, at the borders of $\mathrm{H} 3 \mathrm{~K} 27 \mathrm{me} 3$ domains, short fragment peaks were more frequently associated with CP190, confirming previous reports that this factor is associated with H3K27me3 domain boundaries [24,39] (Fig 2D). Instead, Polycomb was rather enriched at peaks localized internally within these domains. Our Cut\&Run analysis strategy can therefore be used to reveal not only the breadth of histone modification domains in ovaries but also their associated DNA regulatory elements.

\section{dhd lies within an H3K27me3/H3K9me3 mini-domain flanked by DNA regulatory elements}

To gain insight on $d h d$ regulation, we next sought to analyze its associated chromatin configuration. We previously showed that the active transcription modification $\mathrm{H} 3 \mathrm{~K} 4 \mathrm{me} 3$ is enriched at the $d h d$ promoter and that this mark is lost in lid KD ovaries [5] (S2A Fig). Using available ChIP-seq datasets from embryonic derived S2 cells, we further observed that that $d$ hd lies within a $\sim 5 \mathrm{kbp}$ mini-domain featuring two types of repressive histone modifications: H3K27me3 and H3K9me3 (S2A Fig) [40]. H3K27me3 is the hallmark of Polycomb-based repression [41,42], whereas H3K9me3 dictates Heterochromatin Protein 1 (HP1)-based repression $[43,44]$. Interestingly, this mini-domain was also found in ChIP-seq data from fly ovaries (S2A Fig) [45]. Potentially regulating this chromatin environment, Lid and Sin3A were described as participating in a co-repressor complex [20], but their global impact on repressive 
A.

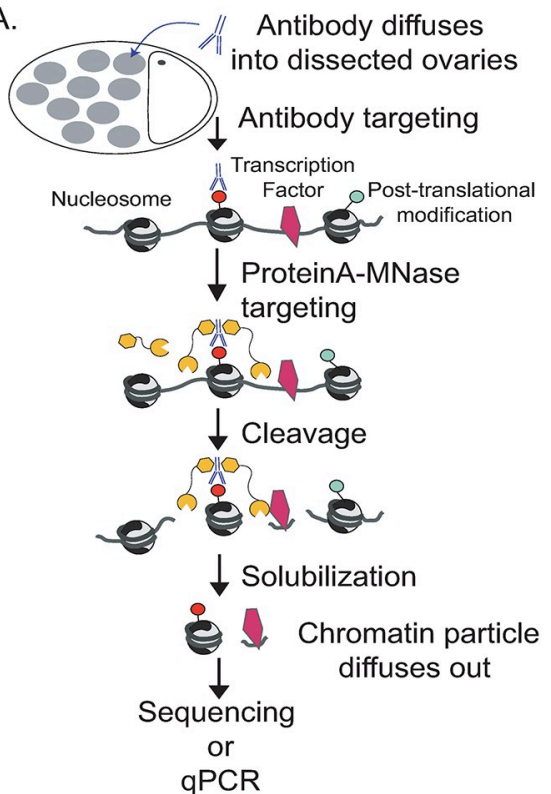

B.

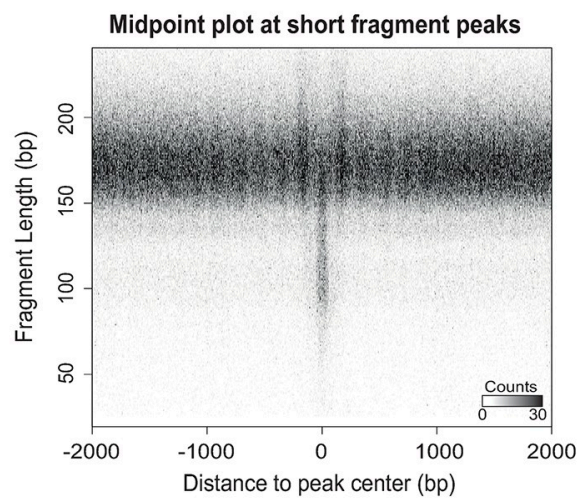

C. ChrsR

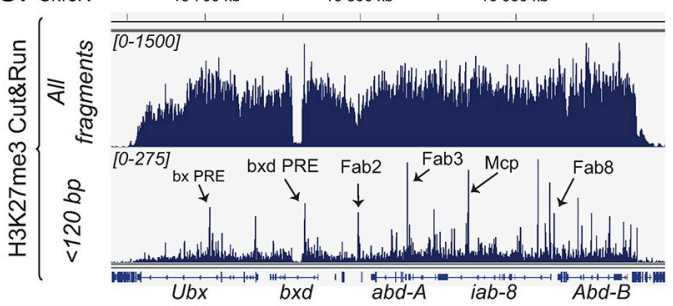

D.
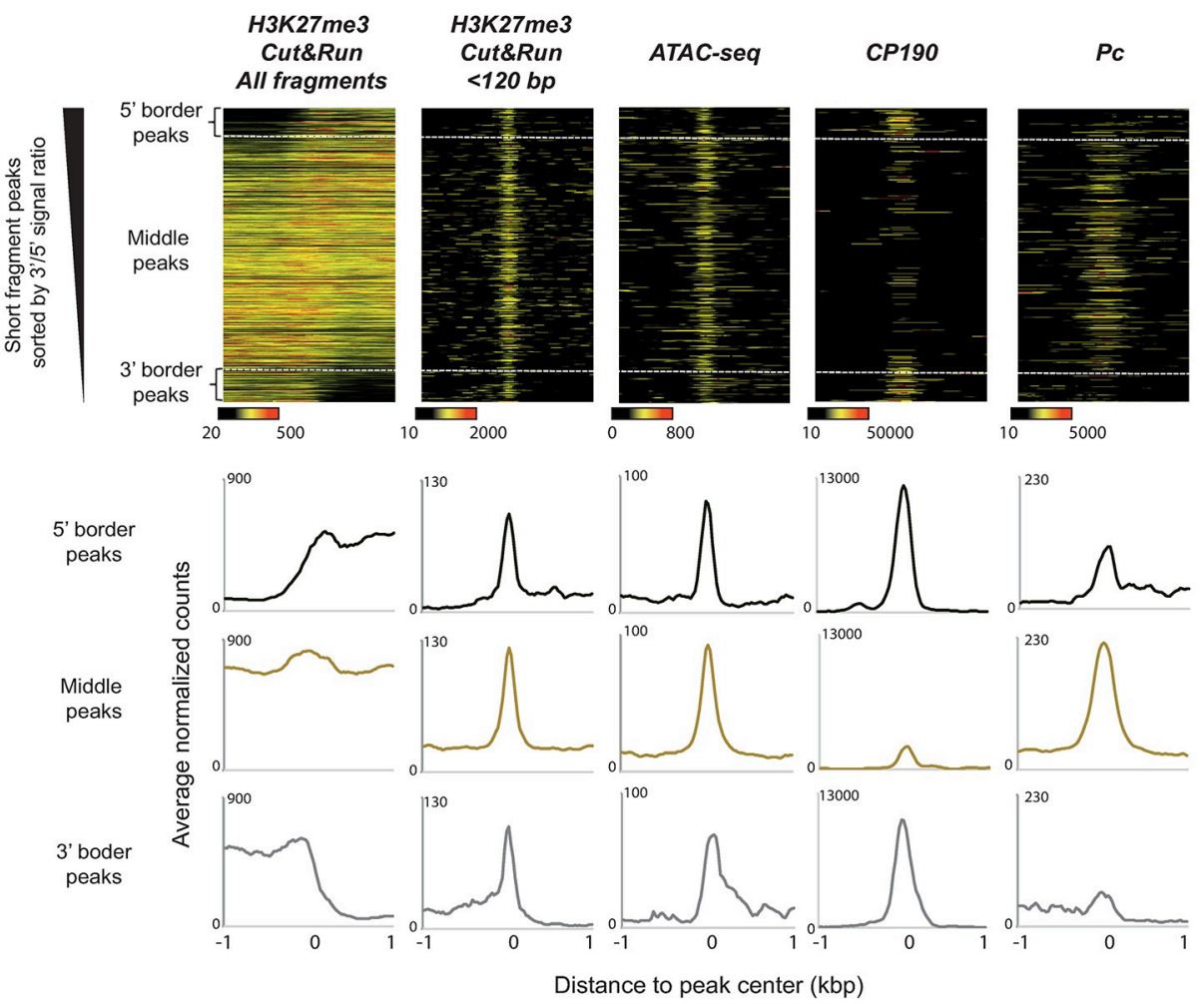

Fig 2. A single Cut\&Run experiment maps both histone modifications and their associated regulatory elements. A -Schematic overview of the Cut\&Run procedure for dissected Drosophila ovaries. After tissue permeabilization and antibody targeting, ProteinA-MNase cleaves nearby exposed DNA allowing the solubilization and retrieval of both nucleosomal particles carrying the targeted histone modification and DNA particles occupied by transcription factors in the immediate vicinity. B-Cut\&Run reveals nucleosomes and transcription factor binding sites. Mid-point plot of ovarian $\mathrm{H} 3 \mathrm{~K} 27 \mathrm{me} 3 \mathrm{Cut} \&$ Run data centered at peaks identified by MACS2 from short fragments $(<120 \mathrm{bp})$ in the same experiment. This plot represents all paired-end sequenced fragments as their middle point coordinate in the X-axis, and their size in the Y-axis, revealing a class of clustered short fragments (50-130bp) flanked by nucleosome-sized fragments (>140bp). C $-\mathrm{H} 3 \mathrm{~K} 27 \mathrm{me} 3$ Cut\&Run at the bithorax complex (BX-C) in Drosophila ovaries reveals its 
regulatory architecture. Genome browser track displaying all Cut\&Run fragments and $<120$ bp fragments separately. Multiple well-described Polycomb Response Elements (PRE) and insulators within the Bithorax complex detected as short fragment peaks are indicated (arrows). D-Cut\&Run re-discovers regulatory elements associated with H3K27me3 genome-wide. Upper panels: short fragment peaks read density heatmaps of ovarian H3K27me3 Cut\&Run (all fragments and $<120 \mathrm{bp}$ fragments), ATAC-seq (from S2 cells, [35]), CP190 ChIP-seq (from Kc cells, [38]) and Polycomb ChIP-seq (Pc, from S2 cells, [37]) plotted at $\pm 1 \mathrm{~kb}$ around peak summit. Data is sorted by the ratio of H3K27me3 Cut\&Run total reads at the 3' versus 5' flanks to reveal short fragment peaks at the borders or within H3K27me3 domains (dashed lines). Lower panels: average profiles corresponding to the top heatmaps, distinguishing 5 ' border peaks, 3 ' border peaks and peaks embedded within domains. Cut\&Run short fragment peaks are enriched for ATAC-seq signal as well as CP190 (particularly at border peaks) and Polycomb (particularly at middle peaks).

https://doi.org/10.1371/journal.pgen.1009615.g002

histone modifications is unclear. In turn, previous reports showed that depletion of insulator proteins $\mathrm{Mod}(\mathrm{mdg} 4)$, as well as CTCF, $\mathrm{Su}(\mathrm{Hw}), \mathrm{CP} 190$ or BEAF-32, did not affect the spread of Polycomb-associated domains but instead caused a general decrease in H3K27me3 levels [24]. In contrast, the Brahma/BAF complex is typically considered as counteracting Polycomb repression based on work in mammals [46], but this interplay has not been analyzed in Drosophila.

Based on these observations, we sought to better characterize the distribution of $\mathrm{H} 3 \mathrm{~K} 27 \mathrm{me} 3$ and H3K9me3 in ovaries at the $d h d$ locus. Using our Cut\&Run approach, we confirmed that dhd is included in a $\sim 5450 \mathrm{bp}$ heterochromatic H3K27me3/H3K9me3 mini-domain that extends from the promoter region of $d h d$ to the promoter of the next gene active in ovaries, Sas10 (Fig 3A). While our whole-tissue Cut\&Run approach cannot distinguish which cells harbor this domain, published $\mathrm{H} 3 \mathrm{~K} 27 \mathrm{me} 3 \mathrm{ChIP}$-seq profiles from either somatic or germline cells in ovaries indicate that this mark is present in both (S2A Fig). We segmented the Cut\&Run H3K27me3 signal in control ovaries and identified 278 discrete H3K27me3 domains, ranging from 3 to $240 \mathrm{~kb}$ in width. The dhd H3K27me3 domain stood out when considering its enrichment in this mark relative to its length, compared to other domains (Fig 3B). Our analyses indicate that despite its reduced size, the $d h d$ locus is capable of accumulating proportionately high amounts of this repressive modification. While we also identified other previously described H3K9me3 domains lodged in euchromatic regions (S3 Fig), the signal is, as expected, largely dominated by pericentric heterochromatin. We could thus not robustly call such euchromatic H3K9me3 domains, preventing us from conducting an analogous analysis for $d h d$ on this mark.

Surprisingly, short fragment peak analysis revealed two putative DNA regulatory elements associated with both histone marks, precisely at the mini-domain borders, with no internal peaks present (Fig 3A). ATAC-seq data from S2 cell lines confirmed presence of only these two border elements (S2B Fig). In addition, in Kc cells, $d$ hd border elements are occupied by CP190 and Mod(mdg4), both of which can be found at the boundaries of Drosophila H3K27me3 domains [24,47]. However, the insulating isoform Mod(mdg4)67.2 is not found at the $d$ hd locus (S2B Fig), suggesting that a different isoform playing an activator function is responsible for $d h d$ regulation. Finally, the $d h d$-proximal 5 ' border element featured a significant, although very modest enrichment for PRE markers Polycomb and Polyhomeotic (S2B Fig). This regulatory architecture was quite unusual, as we could not find any other H3K27me3 domain in the genome sharing this particular organization with two border elements and no internal elements. Together, these results revealed that $d h d$ lies within a unique H3K27me3/H3K9me3 mini-domain featuring only border elements.

\section{Sin3A, Snr1 and Mod(mdg4) control the regulatory architecture of the $d h d$ mini-domain}

We next aimed at evaluating the potential role for Lid, Sin3A, Mod(mdg4) and Snr1 in regulating this heterochromatin mini-domain. We used $\mathrm{KD}$ ovaries for these factors and included as a control a KD for the H3K27 methyltransferase Enhancer of zeste $(\mathrm{E}(\mathrm{z})$ ), induced in germ 
A.

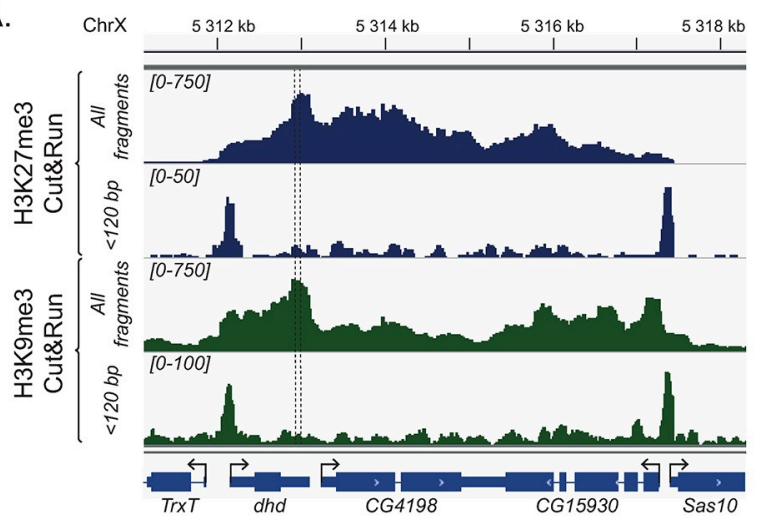

B. H3K27me3 Cut\&Run in ovaries

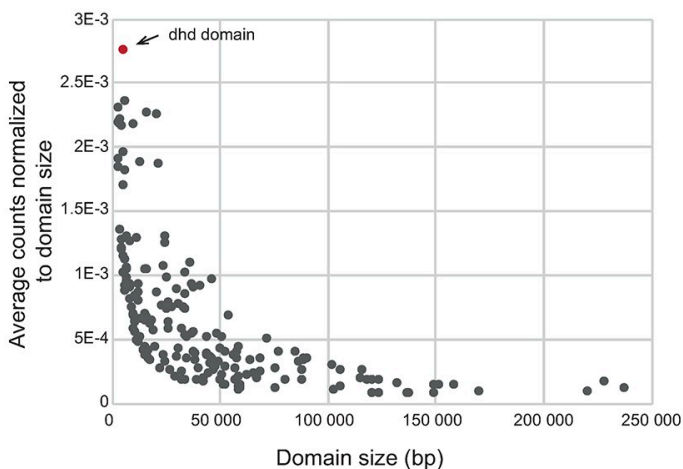

C.

H3K27me3 meta-domains in ovaries

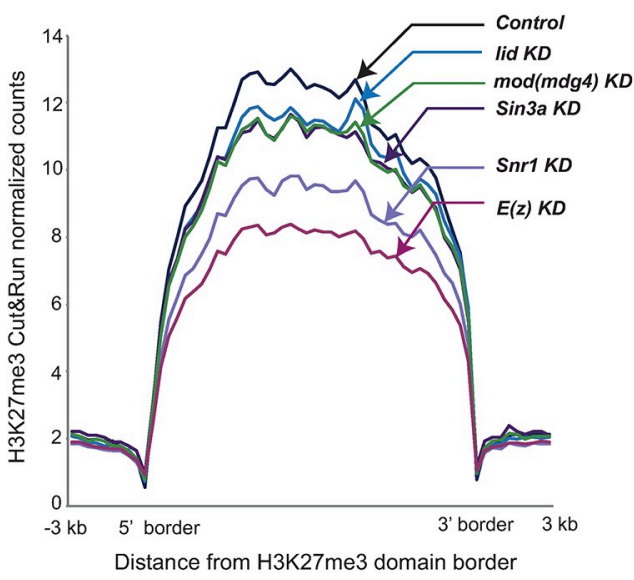

E.

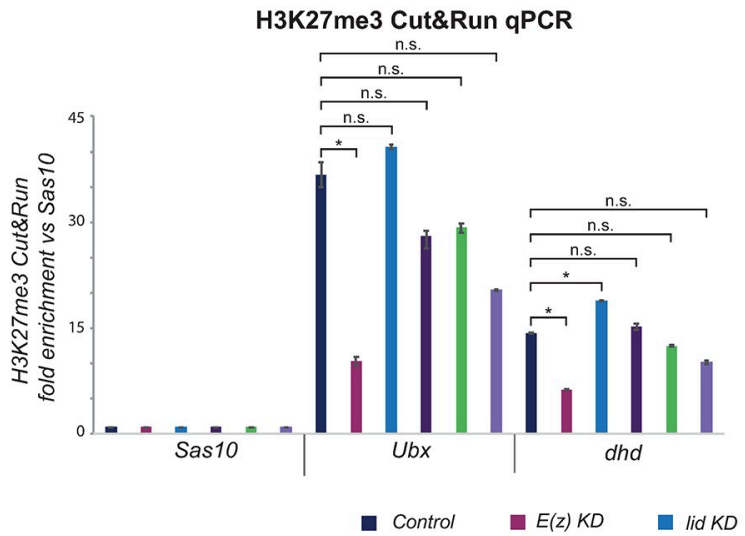

D.

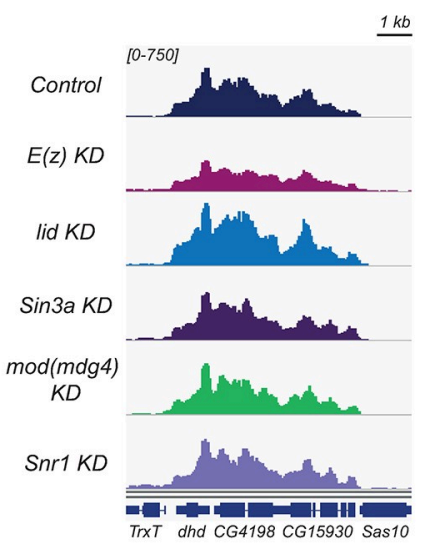

H3K9me3 Cut\&Run qPCR

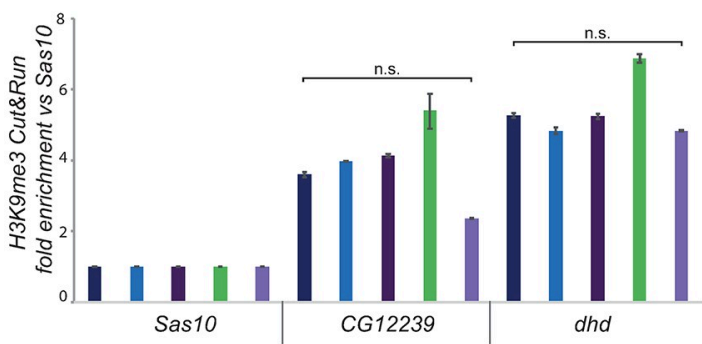

- $\operatorname{Sin} 3 a K D \quad \bmod (\operatorname{mdg} 4) K D \quad \operatorname{Snr} K D$

Fig 3. $d$ h is embedded in an H3K27me3/H3K9me3 mini-domain flanked by regulatory elements. A-The dhd region features an H3K27me3 and H3K9me3 mini-domain. Genome browser snapshots showing the distribution of all fragments and $<120$ bp fragments in the $d h d$ region, revealing that $d h d$ lies within a $\sim 5450 \mathrm{bp}$ heterochromatin mini-domain flanked by border regulatory elements associated with both $\mathrm{H} 3 \mathrm{~K} 27 \mathrm{me} 3$ and $\mathrm{H} 3 \mathrm{~K} 9 \mathrm{me} 3$. Arrows indicate direction of transcription. B-The dhd mini-domain is highly enriched in H3K27me3 relative to its size. Scatter plot of our 278 ovarian H3K27me3 domains identified in Cut\&Run, representing their average read counts normalized to domain size in the $\mathrm{Y}$-axis versus domain size in the $\mathrm{X}$-axis. C-Effect of the KDs on H3K27me3 enrichment genome-wide. Average normalized counts of H3K27me3 Cut\&Run (all fragments) in H3K27me3 domains (plotted as meta-domains and including $\pm 3 \mathrm{~kb}$ from domain borders) in Control $(M T D>+)$, lid $\mathrm{KD}$, Sin $3 a \mathrm{KD}, \bmod (\operatorname{mdg} 4) \mathrm{KD}$, Snr1 KD and $E(z) \mathrm{KD}$ (arrows). D-lid KD, but not Sin3a, Snr1 or mod(mdg4), impacts H3K27me3 enrichment at the dhd mini-domain. Left: genome browser plots of normalized H3K27me3 Cut\&Run signal (all fragments) at the dhd genomic region in Control and KD ovaries. Right: Quantification of 
normalized read counts for the same samples. Data in this figure is for one representative replicate: other replicates are shown in S5A Fig. E- Weak impact of KDs on heterochromatic marks at $d h d$. H3K27me3 and H3K9me3 Cut\&Run-qPCR in Control and KD ovaries using the Sas 10 gene as negative control and $U b x$ and CG12239 as positive controls for H3K27me3 and H3K9me3 respectively. Fold enrichment was calculated relative to Sas10. Error bars show technical variability from a representative replicate. Data in this figure is for one representative replicate: other replicates are shown in S5B Fig. P values indicate one-way ANOVA with Dunnett's multiple comparisons test to a control $\left({ }^{*} \mathrm{P}<0.0001 ; \mathrm{n} . \mathrm{s}=\right.$ not significant $)$.

https://doi.org/10.1371/journal.pgen.1009615.g003

cells by the MTD-Gal4 driver. While $E(z) \mathrm{KD}$ females were sterile as previously described $[48,49]$ (Table 1$)$, they were able to lay eggs and displayed only a moderate effect on $d h d$ expression (a 25\% reduction compared to controls) (S4A Fig). Immunofluorescence staining on dissected control ovaries showed that $\mathrm{H} 3 \mathrm{~K} 27 \mathrm{me} 3$ marks follicle cell nuclei, the karyosome (i.e the oocyte nucleus) and nurse cell nuclei, although nurse cell staining was relatively weaker (S3B Fig), consistent with previous reports [48]. As expected, H3K27me3 was undetectable in the karyosome and in nurse cells of $E(z) \mathrm{KD}$ ovaries, whereas follicle cells (which do not express MTD-driven shRNAs) still carried this mark at normal levels. While lid, Sin3a and mod(mdg4) KD ovaries displayed normal H3K27me3 staining, we observed a moderate reduction in H3K27me3 levels in nurse cells in Snr1 KD ovaries, even while H3K27me3 levels were not affected on the karyosome (S4B Fig).

We next carried out H3K27me3 Cut\&Run on ovaries from all KDs. Within our 278 identified H3K27me3 domains (see above), we compared the average enrichment in H3K27me3 signal in control and $\mathrm{KD}$ ovaries (Fig $3 \mathrm{C}$ ). In $E(z) \mathrm{KD}$ ovaries, Cut\&Run experiments revealed only a moderate loss of $\mathrm{H} 3 \mathrm{~K} 27 \mathrm{me} 3$ signal (35\% average reduction at these domains compared to controls) (Fig 3C), contrasting with the strong global reduction in H3K27me3 immunofluorescence signal. This difference is likely to reflect the fact that the H3K27me3 signal from Cut\&Run experiments originates from both germline and somatic cells. Accordingly, $E(z) \mathrm{KD}$ completely abrogated H3K27me3 signal at the spen, Corto or ptc loci, all of which are decorated with H3K27me3 in nurse cells but not in follicle cells (S4C Fig) [50]. In contrast, the gl, dpp, or repo loci, which show stronger $\mathrm{H} 3 \mathrm{~K} 27 \mathrm{me} 3$ in follicle cells compared to nurse cells, were only slightly affected in $E(z) \mathrm{KD}$ ovaries (S4C Fig). Together, these results show that our Cut\&Run strategy detects H3K27me3 signal from both germline and somatic cells and is able to detect quantitative differences in the averaged signal when nurse cells are strongly affected. Consistent with immunofluorescence experiments, lid, $\operatorname{Sin} 3 a$ and $\bmod (m d g 4)$ KDs had only a modest global impact on average $\mathrm{H} 3 \mathrm{~K} 27 \mathrm{me} 3$ levels (5\% reduction compared to control), and no effect on the spread of H3K27me3 domains (Fig 3C). Also consistent with our immunofluorescence experiments, Snr1 KD led to a more severe average reduction of H3K27me3 Cut\&Run signal compared to control (20\%), although not as dramatic as $E(z) \mathrm{KD}$.

In agreement with genome-wide observations, the levels of $\mathrm{H} 3 \mathrm{~K} 27 \mathrm{me} 3$ in the $d h d$ minidomain were reduced in $E(z) \mathrm{KD}$ ovaries and unaffected in $\operatorname{Sin} 3 a$ or $\bmod (\operatorname{mdg} 4) \mathrm{KD}$ ovaries. More surprisingly, the domain was not measurably affected in Snr1 KD ovaries, despite the fact that H3K27me3 is globally impacted by this knockdown (Figs 3D and S5A). Within the sensitivity limits of our approach, these results indicate that $\operatorname{Sin} 3 a$, $\operatorname{Snr} 1$ and $\bmod (\operatorname{mdg} 4)$ KDs have little if any impact on $\mathrm{H} 3 \mathrm{~K} 27 \mathrm{me} 3$ at the $d$ hd locus. Conversely, in lid $\mathrm{KD}$ ovaries, in which global H3K27me3 levels were unaffected, we detected an increase in H3K27me3 levels at the $d h d$ mini-domain (Figs 3C, 3D and S5A). This raised the possibility that Lid could facilitate $d h d$ expression by counteracting Polycomb-mediated repression.

Since the $d h d$ mini-domain also featured H3K9me3, we next turned to Cut\&Run followed by qPCR to evaluate its status in KD ovaries. H3K27me3 Cut\&Run-qPCR measures the expected enrichments at $\mathrm{H} 3 \mathrm{~K} 27 \mathrm{me} 3$ domains and detects variations in the signal coherent with Cut\&Run-seq results (Figs 3E and S5B). To validate the H3K9me3 Cut\&Run-qPCR 
approach in ovaries, we exploited the CG12239 gene as a positive control [45], and detected an expected enrichment in $\mathrm{H} 3 \mathrm{~K} 9 \mathrm{me} 3$ signal at this locus (Figs 3E and $\mathrm{S} 5 \mathrm{~B}$ ). At the dhd locus, $\mathrm{H} 3 \mathrm{~K} 9 \mathrm{me} 3$ was enriched as expected from ChIP-seq results. Importantly, knockdown of lid, $\operatorname{Sin} 3 a, \bmod (m d g 4)$ or Snr1 had no effect on this enrichment (Fig 3E). The $d h d$ heterochromatin mini-domain including H3K27me3 and H3K9me3 is thus independent of Sin3A, Snr1, whereas Lid counteracts H3K27me3.

We next evaluated the impact of different KDs on the $d h d$ mini-domain short fragment peaks at border regions. We first analyzed the effect of our different knockdowns on the full set of 679 peaks previously defined (Fig 2B). Both $E(z)$ and $S n r 1 \mathrm{KD}$ led to a strong ( 63\%) decrease in short fragment peak average counts genome-wide (Fig 4A). Since these KDs also affect global H3K27me3 levels, this reduction could result from a general absence of histone modification-targeted MNase on chromatin. Remarkably, Sin $3 a$ KD led to a similarly strong effect on short fragment peak counts that could not be attributed to its global impact on H3K27me3. Instead, this data suggests that Sin $3 \mathrm{~A}$ is required to ensure proper occupancy and organization of transcription factors and/or nucleosomes at DNA regulatory elements associated with $\mathrm{H} 3 \mathrm{~K} 27 \mathrm{me}$. In contrast, lid or $\bmod (m d g 4) \mathrm{KD}$ did not globally affect short fragment peak counts, indicating that these factors do not play such a role (Fig 4A).

Consistent with their effects genome-wide, short fragment counts at the $d h d$ mini-domain border elements were strongly diminished upon $E(z)$ and $\operatorname{Sin} 3 a$ KDs (Figs 4B and S5C). Intriguingly, $\bmod (m d g 4) \mathrm{KD}$ led to a similar impact on these border elements (particularly the dhd-proximal one), even though it did not globally affect H3K27me3-associated elements genome-wide (Figs 4B and S5C). This observation could indicate that the $d$ hd border elements become less frequently occupied by transcription factors, that these factors become less frequently associated with $\mathrm{H} 3 \mathrm{~K} 27 \mathrm{me} 3$, and/or that their nucleosomal organization is compromised. In all cases, this suggests that $\operatorname{Mod}(\operatorname{mdg} 4)$ is required to ensure chromatin organization of the border DNA regulatory elements at the $d h d$ mini-domain. Remarkably, Snrl KD led to a similar effect on border elements without affecting H3K27me3 levels at the $d h d$ minidomain, suggesting that Snr1 is also required for the proper organization of the $d h d$ border elements. In striking contrast, lid $\mathrm{KD}$ had no detectable effect on these regulatory elements (Figs $4 \mathrm{~B}$ and $\mathrm{S} 5 \mathrm{C}$ ). We concluded that Lid, although essential for $d h d$ expression, was not required to ensure the proper organization of $d h d$ border elements.

Altogether our results, summarized in Fig 4C, indicate that Lid, Sin3A, Snr1 and Mod (mdg4), impact H3K27me3 or its associated regulatory elements genome-wide and/or at the dhd mini-domain in four distinct manners.

\section{The $d h d$ promoter-proximal DNA regulatory element is required for $d h d$ expression independently of its heterochromatin mini-domain}

We next performed sequence analysis of the $d h d$ mini-domain border elements, screening against the flyreg.v2 transcription factor DNA binding motif database [51,52]. At the 5 ' border element, which mapped to the $d h d$ promoter region, we identified four perfect matches for the DNA replication-related element (DRE) motif, TATCGATA (Fig 5A). This motif is recognized by the insulator-associated factor BEAF-32 [53] and the core-promoter factor DREF [16]. These four DRE motifs overlap in the palindromic sequence TATCGATATCGATA, 37bp upstream of the $d h d$ transcription start site. Consistently, BEAF-32 and DREF both occupy this element in Kc cells (S6 Fig) [38]. Previous studies showed that BEAF-32 null females are partially fertile ( $\sim 40 \%$ hatching rate) [54], indicating that this factor is not essential for $d h d$ expression. In turn, DREF is essential in a cell-autonomous manner and indeed dref mutations cause oogenesis defects [55]. Accordingly, we observed severe atrophy and failure to produce 
A. H3K27me3-associated regulatory elements in ovaries

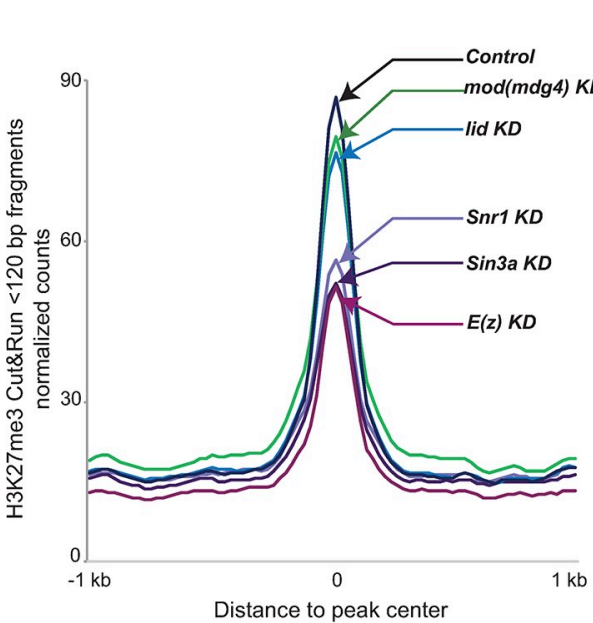

B. H3K27me3-associated regulatory elements at the dhd locus

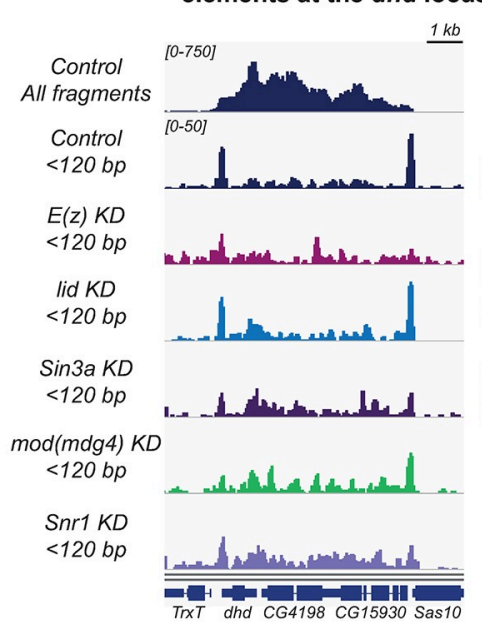

Control

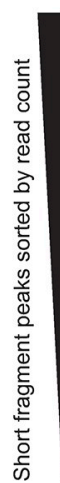

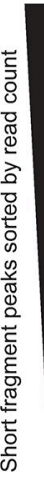
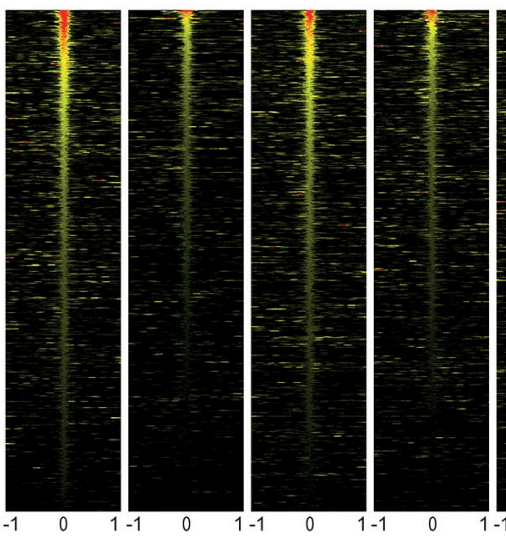

$\bmod (\operatorname{mdg})$ Snr1 KD

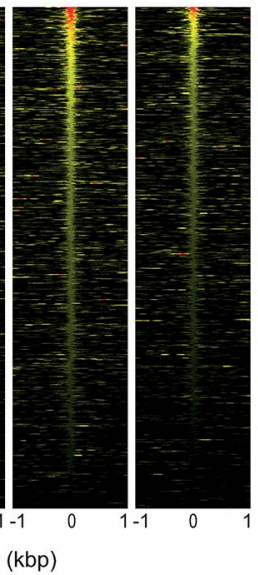

Distance to peak center (kbp)

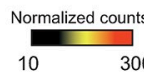

C.

\begin{tabular}{|c|c|c|c|c|}
\hline $\begin{array}{l}\text { Effect vs } \\
\text { Control }\end{array}$ & $\begin{array}{c}\text { Genome-wide } \\
\text { H3K27me3 }\end{array}$ & $\begin{array}{l}\text { dhd domain } \\
\text { H3K27me3 }\end{array}$ & $\begin{array}{c}\text { Genome-wide } \\
\text { H3K27me3- } \\
\text { RE }\end{array}$ & $\begin{array}{l}\text { dhd domain } \\
\text { H3K27me3- } \\
\text { RE }\end{array}$ \\
\hline$E(z) K D$ & $y$ & ע & ? & ? \\
\hline lid KD & $\approx$ & $\pi$ & $\approx$ & $\approx$ \\
\hline $\operatorname{Sin} 3 a K D$ & $\approx$ & $\approx$ & y & y \\
\hline $\begin{array}{c}\bmod (\operatorname{mdg} 4) \\
K D\end{array}$ & $\approx$ & $\approx$ & $\approx$ & ע \\
\hline Snr1 KD & ע & $\approx$ & ? & ע \\
\hline
\end{tabular}

Fig 4. Sin 3A, Snr1 and Mod(mdg4) control the regulatory architecture of the $d h d$ H3K27me3 mini-domain. A-Sin $3 a$ and Snr1 KD, but not lid or $\bmod (m d g 4)$, impact H3K27me3-associated regulatory elements genome-wide. Left: H3K27me3 Cut\&Run $<120$ bp fragments normalized counts in Control and KD ovaries, plotted at $\pm 1 \mathrm{~kb}$ around the summit of short fragment peaks. Right: Heatmaps displaying H3K27me3 Cut\&Run short fragment peaks normalized read counts $\pm 1 \mathrm{~kb}$ around peak center in Control and KD ovaries. BSin3A, Snr1 and Mod(mdg4), but not Lid, impact the organization of regulatory elements at the borders of the dhd H3K27me3 minidomain. Left: genome browser plots of normalized Control H3K27me3 Cut\&Run signal (all fragments, top) and of normalized signal from < 120bp fragments retrieved in H3K27me3 Cut\&Run in Control and KD ovaries. Right: Quantification of $<120 \mathrm{bp}$ fragments normalized read counts for the same samples. 5' and 3' border elements are plotted separately. Data in this figure is for one representative replicate: other replicates are shown in S4C Fig. Table recapitulating the effect of the different KDs on H3K27me3 and H3K27me3-associated regulatory elements (H3K27me3-RE) genome-wide and at the $d h d$ locus. " $\approx$ " indicates modest or no change, " $\nearrow$ " indicates an increase and " \" a decrease in average read counts compared to Control. "?" indicates inability to conclude.

https://doi.org/10.1371/journal.pgen.1009615.g004

oocytes in dref KD ovaries. Because this precluded studying the role of DREF in dhd regulation, we instead sought to probe the importance of the DRE motifs themselves.

The $d h d^{55}$ null allele is a $1.4 \mathrm{~kb}$ deletion affecting the entire promoter region including the promoter-proximal regulatory element, and part of the coding region of $d h d$ [7,9] (Fig 5A). A $p W 8-d h d^{W T}$ transgenic construct, bearing the entire $d h d$ gene -including its promoter region-, restores $d h d$ expression as well as fertility in $d h d^{J 5}$ mutants [9] (Fig 5A, 5B and Table 1). We 
A.
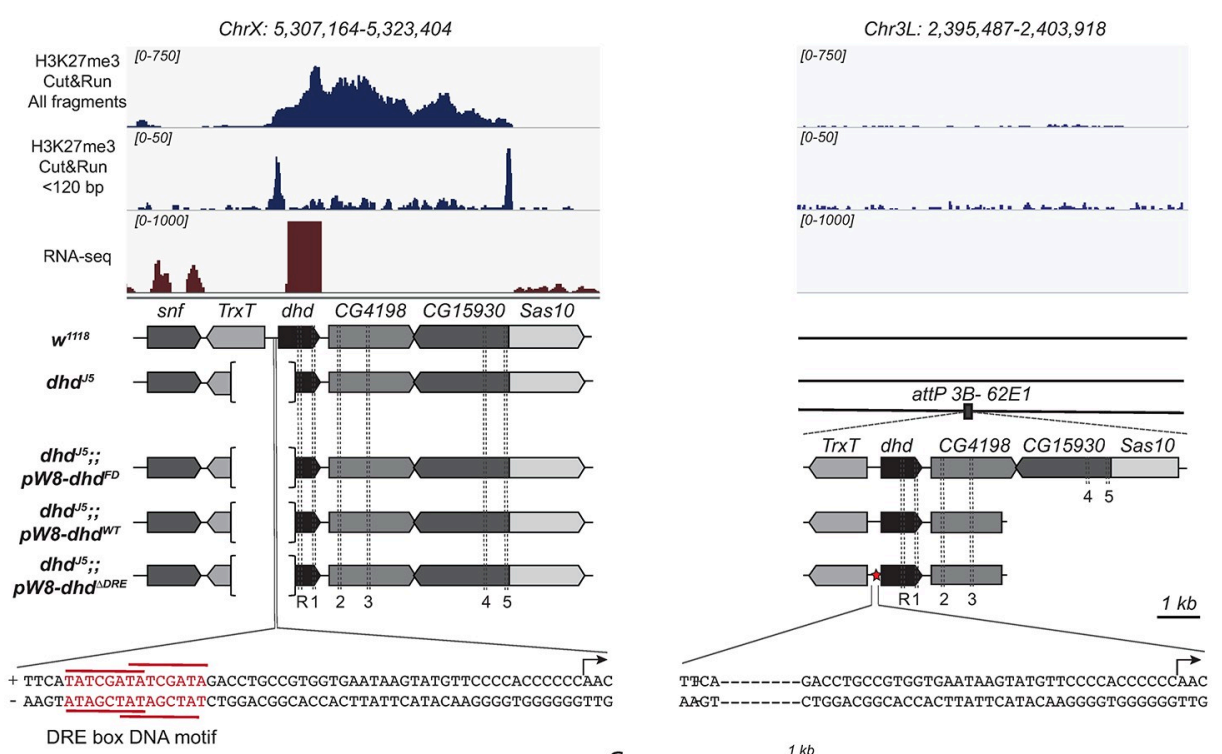

B. DRE box DNA motif

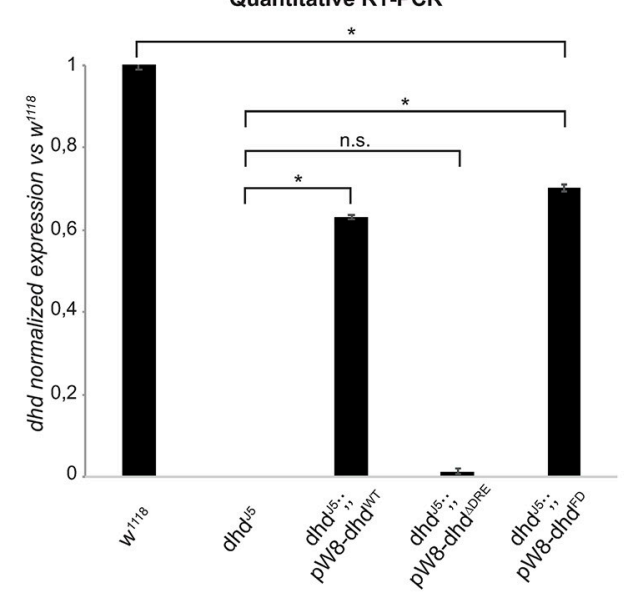

C.
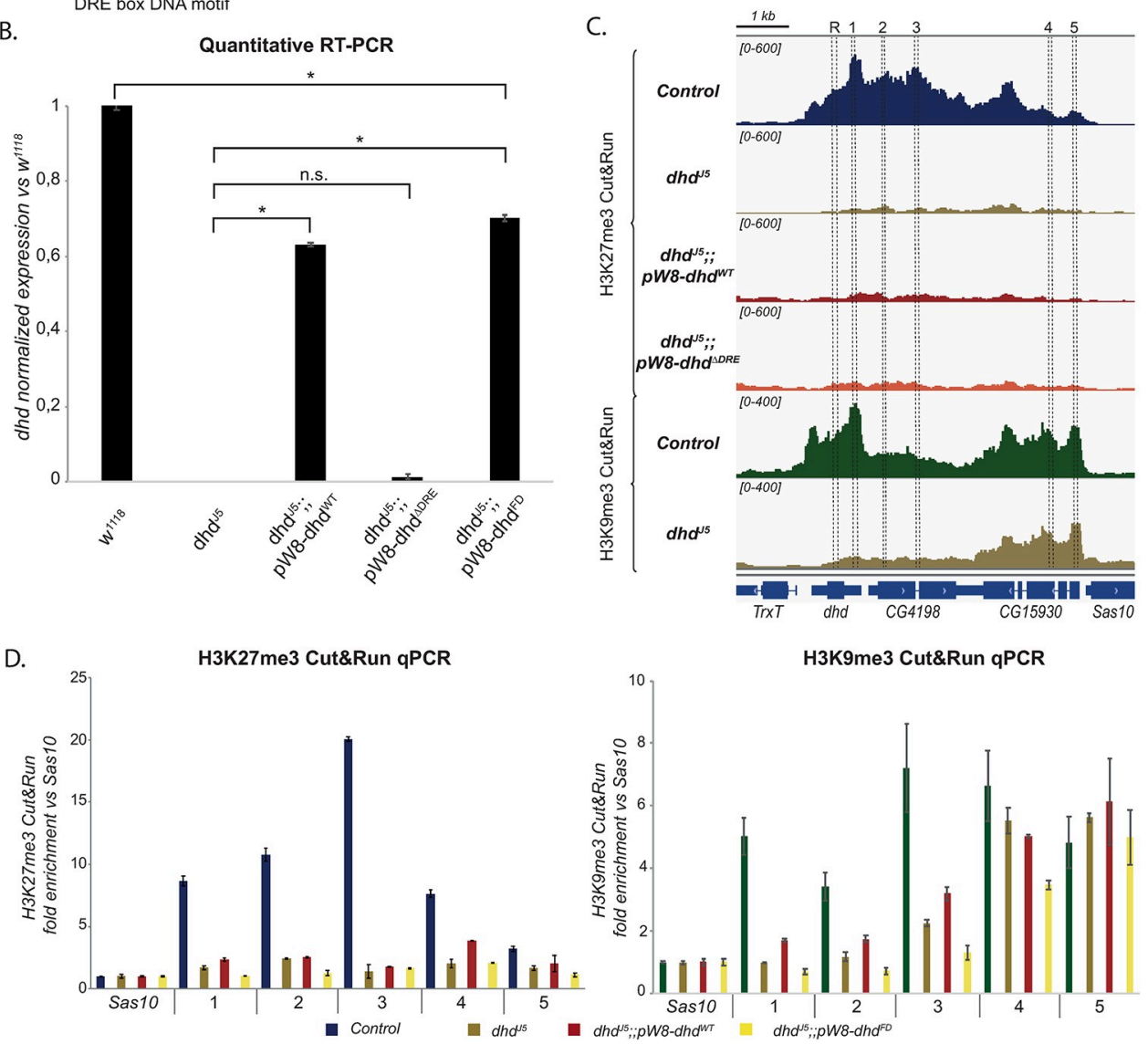

Fig 5. The $d h d$ promoter-proximal DRE motifs are required for its expression. A-Schematic representation of the genotypes studied in this figure. Upper panels: genomic browser views recapitulating the Control distribution of H3K27me3 Cut\&Run signal (all fragments and $<120 \mathrm{bp}$ fragments) as well as RNA-seq signal from Figs 1 and 2 at the $d h d$ locus and showing lack of signal at the transgene insertion locus in the absence of any transgenic construct. Middle panel: schematic representation of the genomic composition of $w^{1118}$ (reference strain), mutant and rescue flies, indicating the status of the $d h d$ locus and the composition of the rescue transgene. Dashed lines indicate the targeted region by primer couples (primers R and 1-5) used for RT- and Cut\&Run-qPCR in panels B and D. Bottom panel: sequence of the $d h d$ promoter at the endogenous location (left) and in the $\triangle \mathrm{DRE}$ mutant transgene where the $14 \mathrm{bp}$ containing the DRE motifs were deleted. B- The DRE motifs at the $d h d$ promoter are necessary for its expression. 
RT-qPCR quantification of $d h d$ mRNA levels in ovaries from wild-type $w^{1118}$ flies, $d h d^{55}$ mutants or $d h d^{55}$ mutants carrying either a WT $\left(p W 8-d h d^{W T}\right)$ or a mutant $\left(p W 8-d h d^{A D R E}\right)$ or a full domain $\left(p W 8-d h d^{F D}\right)$ transgene (measured using the R primers, normalized to $r p 49$ and relative to expression in $w^{1118}$ ). Data from biological duplicates analyzed in technical duplicates are presented as mean \pm SEM. P values indicate one-way ANOVA with Tukey's multiple comparisons test $\left({ }^{*} \mathrm{P}<0.0001 ; \mathrm{n} . \mathrm{s}=\right.$ not significant). C- The $d h d$ heterochromatin domain is affected in $d h d-$ containing transgenic constructs. Genome browser plots of normalized ovarian H3K27me3 and H3K9me3 Cut\&Run signal at the dhd genomic region in the indicated genotypes. The H3K27me3 domain is abolished in all transgenic rescues. The H3K9me3 domain is partly affected in the $d h d^{j 5}$ mutant, being lost the $d h d$-proximal end but maintained in the $d h d$-distal end. Dashed lines and numbers ( 1 to 5 ) indicate the targeted region by primer couples used for qPCR in panel D. D- A transgene containing the full $d h d$ domain does not restore heterochromatin marks. H3K27me3 and H3K9me3 Cut\&Run-qPCR in Control, $d h d^{J 5}, d h d^{75} ; ; p W 8-d h d^{W T}$ and $d h d^{75} ; ; p W 8-d h d^{F D}$ ovaries. Fold enrichment was calculated relative to Sas 10. Error bars show technical variability from a representative replicate. Data in this figure is for one representative replicate: other replicates are shown in S7 Fig.

https://doi.org/10.1371/journal.pgen.1009615.g005

now constructed a second rescue transgene based on the $p W 8-d h d^{W T}$, where the $14 \mathrm{bp}$ carrying the DRE motifs were deleted ( $p W 8$ - $d h d^{\Delta D R E}$ ) (Fig 5A). These constructs were inserted into the same genomic location as $p W 8-d h d^{W T}$ (62E1) and combined with the $d h d^{J 5}$ deficiency. In striking contrast to $p W 8-d h d^{W T}$, the $p W 8-d h d^{\Delta D R E}$ construct was unable to rescue $d h d$ expression, or substantially improve fertility in $d h d^{J 5}$ deficient flies (Fig 5B and Table 1). The DRE motifs are thus essential to ensure $d h d$ expression.

To test a role for this regulatory element and its DRE motifs in regulating the H3K27me3/ H3K9me3 mini-domain, we performed Cut\&Run-seq and Cut\&Run-qPCR on homozygous $d h d^{J 5}$ ovaries, as well as rescue $d h d^{J 5} ; p W 8-d h d^{W T}$ and non-rescued $d h d^{J 5} ; ; p W 8-d h d^{\Delta D R E}$ ovaries. Strikingly, the 5.4kbp $d h d \mathrm{H} 3 \mathrm{~K} 27 \mathrm{me} 3$ mini-domain was completely lost in $d h d^{15}$ ovaries (Figs 5C and 5D and S7), despite the fact that 90\% of this mini-domain were intact in the deficient chromosome. This indicates that the $d h d$-proximal border of this mini-domain is essential for establishment and/or maintenance of H3K27me3. Furthermore, H3K27me3 signal was absent within the mini-domain in $d h d^{J 5} ; ; p W 8-d h d^{W T}$ rescue ovaries (Figs 5C and 5D and S7), suggesting that the 5 '-most $2.8 \mathrm{kbp}$ of the domain are also insufficient to establish and/or maintain H3K27me3. This result further confirms that $d h d$ can be expressed at high levels in the absence of H3K27me3, consistent with results from $E(z) \mathrm{KD}$ ovaries (S4A Fig). Finally, the H3K27me3 mini-domain was also completely absent in $d h d^{J 5} ; p W 8-d h d^{\Delta D R E}$ ovaries (Fig 5C), indicating that the DRE motifs are required for $d h d$ expression independently of H3K27me3.

Based on these observations, we hypothesized that the complete mini-domain sequence, including both border regulatory elements, might be necessary for restoring heterochromatin marks. We thus constructed a transgene containing the full domain sequence of the $d h d$ heterochromatin domain ( $p W 8-d h d^{F D}$ ) (Fig $5 \mathrm{~A}$ ), inserted at the same genomic location as the $p W 8-d h d^{W T}$ transgene. Interestingly, this transgene restored $d h d$ expression (Fig 5B) and rescued fertility (Table 1) but was unable to restore H3K27me3 (Figs 5D and S7). These results indicate that the border-to-border mini-domain is not autonomous and suggest that its genomic location impacts its chromatin configuration.

We next focused on the H3K9me3 mark. In contrast to H3K27me3, Cut\&Run analysis in $d h d^{55}$ mutants showed that $\mathrm{H} 3 \mathrm{~K} 9 \mathrm{me} 3$ was lost at the $d h d$-proximal half of the domain, while this mark was maintained at the $d h d$-distal part (Fig 5C). Cut\&Run-qPCR using primers across the $d h d$ domain revealed that $\mathrm{H} 3 \mathrm{~K} 9 \mathrm{me} 3$ was not restored at the $d h d$-proximal part of the H3K9me3 domain in $d h d^{55} ;$; pW8- $d h d^{W T}$ or $d h d^{55} ; ; p W 8-d h d^{F D}$ ovaries (Figs 5D and S7). Together, these results indicate that the border-to-border mini-domain is not autonomous to establish its own heterochromatin configuration, and that $d h d$ expression can proceed at near normal levels independently of these marks. 


\section{Lid, Sin3A, Snr1 and Mod(mdg4) activate $d h d$ independently of its heterochromatin mini-domain}

The fact that the $p W 8-d h d^{W T}$ transgene restored most of $d h d$ expression without re-establishment of the heterochromatin mini-domain at this locus provided an opportunity to clarify the role of our set of $d h d$ regulators. KD of lid is associated with increased H3K27me3 at the $d h d$ mini-domain, suggesting that Lid may operate as an anti-repressor by counteracting heterochromatinization of the locus. However, we have previously found that $d$ hd expression is not re-established in lid KD ovaries carrying a $p W 8-d h d^{W T}$ rescue transgene [5]. Lid is thus required for $d h d$ expression not only at its endogenous locus but also from the rescue transgene not decorated by H3K27me3 (Fig 5). Therefore, Lid activates $d$ hd independently of heterochromatin, suggesting that it does not operate strictly as an anti-repressor.

To discriminate between anti-repressive or activating roles of Sin3A, Mod(mdg4) and Snr1, we generated flies combining a $d h d^{J 5}$ deficiency, the $p W 8-d h d^{W T}$ transgene and an shRNA targeting lid, Sin3a, Snr1 or mod(mdg4), driven in germ cells by a nos-Gal4 driver (Fig 6A). We confirmed by RT-qPCR that knockdowns were still efficient when using this driver (S8A Fig). Remarkably, all of these flies were almost completely sterile, and showed strong downregulation of $d h d$ revealed by RT-qPCR (Fig 6B and Table 1). Using Cut\&Run-qPCR at the $d h d$ locus, we further confirmed that these knockdowns had no effect on H3K27me3, which remained depleted in all conditions (Figs 6C and S8B). Lid, Sin3A, Snr1 and Mod(mdg4) therefore stimulate $d h d$ transcription in the absence of its heterochromatin mini-domain.

Our results indicate that the $d h d$ heterochromatin mini-domain does not play a repressive role in ovaries, but do not exclude that it might maintain $d h d$ silent in other tissues. RT-qPCR analysis on dissected ovaries, testes and male and female carcasses from transgenic lines expressing $d h d^{J 5} ;$; $W 8-d h d^{W T}$ revealed $d h d$ expression uniquely from ovaries (Fig 6D).

Because this transgene rescues $d h d$ expression without restoring heterochromatin marks, these results suggest that the $d h d$ heterochromatin mini-domain is not essential to repress ectopic $d h d$ expression in adults. We note, however, that we cannot exclude that $d h d$ was weakly and/ or transiently expressed in certain cell types in these conditions, or that the rescue transgene could accumulate repressive marks in tissues other than ovaries.

\section{Discussion}

\section{The ovarian hyperactivation of $d h d$}

Here, we sought to understand how the genomic and epigenomic environments of $d h d$ contributed to its remarkable regulation, its expression being both among the highest in Drosophila, and absolutely specific to adult ovaries [5,9]. Lid, Sin3A, Snr1 and Mod(mdg4) all shared a critical and rather specific role in ensuring $d h d$ expression. Yet, these four broadly expressed proteins play multiple roles other than $d h d$ regulation. For example, transcriptomic analyses following individual depletion of Lid, Sin 3A or Snr1 in S2 cells, wing discs or pupae shows activation or repression of hundreds of targets $[19,22,56]$. ChIP-seq data further indicates that $\operatorname{Mod}(\operatorname{mdg} 4)$, Sin3A and Lid each target several thousand sites in the genome [24,26,57,58]. Consistently, our RNA-seq analyses did reveal that each of these knockdowns were associated to up- or down-regulation of 407 to 2020 genes in ovaries, with $d h d$ being in every case among the most strongly dependent on these factors. We propose that $d h d$ is a hypersensitive gene that reacts radically to epigenome imbalances.

The key question is therefore what is the formula for $d h d$ ovarian hyperactivation. One reasonable hypothesis was that $d h d$ could be highly regulated by distal enhancers. This would be notably consistent with the previously described role of $\operatorname{Mod}(\operatorname{mdg} 4)$ in organizing 3D contacts 
A.

dhd locus

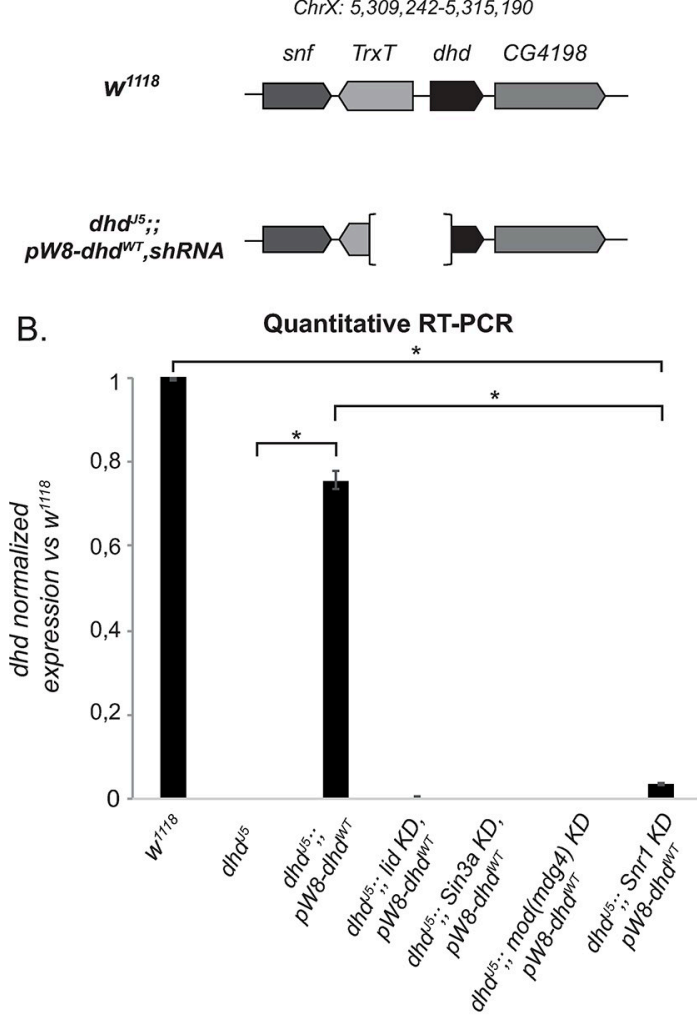

dhd rescue transgene

Chr3L: 2,395,487-2,399,793

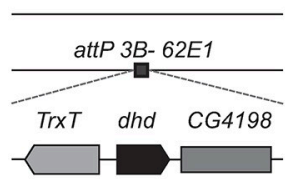

ShRNA under UAS-Gal4 control

Chr3L: 11,070,538- 11,071,193

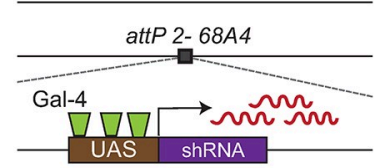

C.

H3K27me3 Cut\&Run qPCR

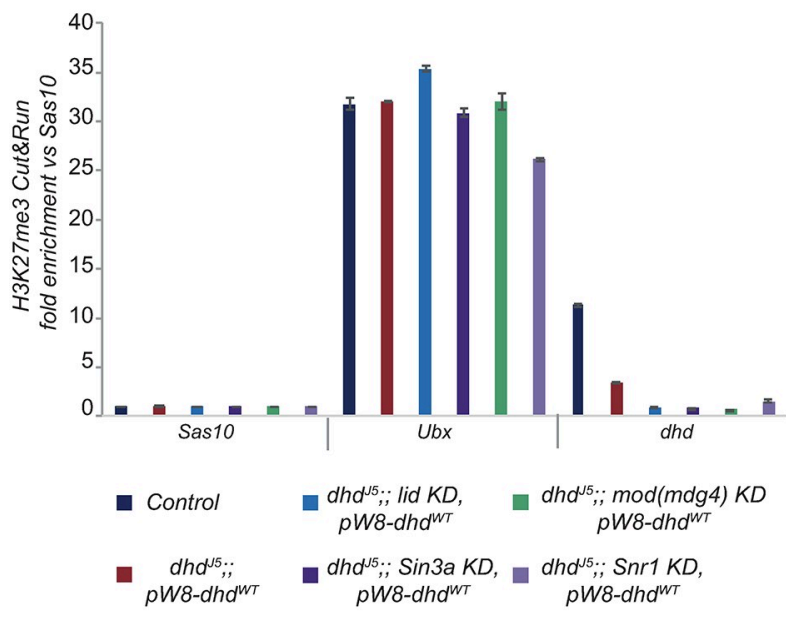

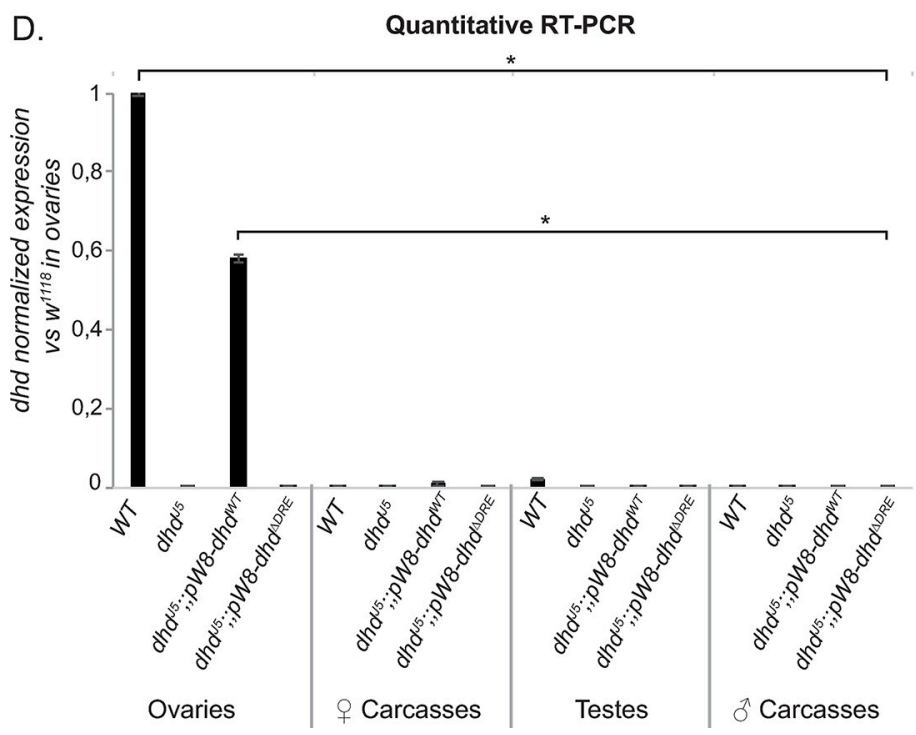

Fig 6. Lid, Sin3A, Mod(mdg4) and Snr1 are necessary for $d h d$ expression in the absence of its heterochromatin domain. ASchematic representation of the genomic composition of $w^{1118}$ (reference strain) and mutant flies carrying a rescue transgene and shRNA constructs controlled by the female germline specific nanos-Gal4 driver, respectively inserted at the platforms attP $3 B-62 E 1$ and attP 2-68A4. B-The rescue transgene does not restore $d h d$ expression in KD ovaries. RT-qPCR quantification of $d h d$ mRNA levels in ovaries of the indicated genotypes (normalized to $r p 49$ and relative to expression in $w^{1118}$ ovaries). Data from biological duplicates analyzed in technical duplicates are presented as mean \pm SEM. P value indicates one-way ANOVA with Tukey's multiple comparisons test ( $\left.{ }^{*} \mathrm{P}<0.0001\right), \mathrm{C}-\mathrm{H} 3 \mathrm{~K} 27 \mathrm{me} 3$ is absent from the $d h d$ rescue transgene. H3K27me3 Cut\&Run-qPCR in the indicated genotypes. The Sas 10 gene was used as negative control and $U b x$ as positive control. Fold enrichment was calculated relative to Sas10. Error bars show technical variability from a representative replicate. Error bars show technical variability from a representative replicate. Data in this figure is for one representative replicate: other replicates are shown in S8 Fig. D- $d$ hd is not ectopically expressed in adult tissues in the absence of its heterochromatin domain. RT-qPCR quantification of $d h d$ mRNA levels in dissected ovaries or corresponding female 
carcasses as well as testes or corresponding male carcasses, in all indicated genotypes (normalized to $r p 49$ and relative to expression in ovaries in $w^{1118}$ ). Data from biological duplicates analyzed in technical duplicates are presented as mean \pm SEM. P value indicates oneway ANOVA with Tukey's multiple comparisons test $\left({ }^{*} \mathrm{P}<0.0001\right)$.

https://doi.org/10.1371/journal.pgen.1009615.g006

between regulatory elements and promoters [26]. It would also be consistent with recent findings that H3K27me3 micro-domains may reflect such contacts [59]. However, no interaction between $d h d$ and any other locus can be found in $\mathrm{Hi}-\mathrm{C}$ data, and our rescue transgene experiments show that a small, ectopic genomic segment almost fully recapitulates its expression, arguing that the genomic and epigenomic environment at the endogenous $d h d$ region may play only a minor role in its ovary-specific hyperactivation.

We indeed found a key regulatory element containing a tandem DRE motif, known to recruit the DREF core promoter factor. The minimal DRE motif (TATCGATA) is found in thousands of gene promoters [60], while multiple genes were individually shown to require this motif for proper activation. These include genes with ovarian expression, and, accordingly, DREF mutations cause oogenesis defects and female sterility [55]. In contrast, the particular tandem DRE motif in the $d h d$ regulatory sequence is uncommon, being only found in 9 other gene promoters. Yet, among these 9 genes, only 4 displayed an expression bias in ovaries, and none were nearly as highly transcribed as $d h d$. Therefore, this motif does not seem to be autonomously sufficient for ovarian hyperexpression.

Another unusual feature of $d h d$ is its surrounding heterochromatin mini-domain bearing both H3K27me3 and H3K9me3 marks, as well as H3K4me3. The co-occurrence of these active and repressive modifications at an ensemble of developmentally regulated genes in mammals led to the concept of bivalent promoters [61]. It is speculated that such promoters may be poised for rapid activation or repression upon differentiation. In Drosophila, bivalent chromatin is associated with genes that can be strongly activated in a tissue-specific manner $[62,63]$. Our experiments showed that $d h d$ is expressed at $\sim 60-70 \%$ of its normal levels in $E(z) \mathrm{KD}$ ovaries, as well as in rescue transgenes-conditions in which the H3K27me3/H3K9me3 minidomain is impaired. We thus cannot exclude that these heterochromatin marks play a positive role in $d h d$ activation to ensure its transcription at maximum capacity, perhaps via establishment of a bivalent configuration. We also note that our whole-tissue experiments leave the possibility open that these histone modifications may decorate $d h d$ in different cell types and/ or at different times during gametogenesis.

Altogether, we uncovered multiple unusual genomic and epigenomic characteristics at the $d h d$ locus, but failed to identify any single feature that was truly defining. The dramatic regulation of $d h d$ may rely not on any individual trait but rather on a unique combination of such rare features. Further work will be needed to elucidate how these different components may together achieve ovarian hyperexpression.

\section{A non-canonical chromatin domain}

The unique properties of $d h d$ led us to uncover interesting features of its epigenomic regulators. First, $d h d$ is embedded in an H3K27me3 mini-domain flanked by regulatory elements. A recent report suggested that $\mathrm{H} 3 \mathrm{~K} 27 \mathrm{me} 3$ domain borders may be established independently of PREs or border elements, provided that an immediately neighboring active gene instead delimits H3K27me3 spreading [47]. The case of $d h d$ is however peculiar in that the H3K27me3 domain border overlaps with this highly active gene, a scenario that was not found in other domains. The coincidence of H3K27me3 and H3K9me3 is also uncommon and, in fact, we could not find any other such dual domain in ovaries. Our result favors the view that this heterochromatin domain does not silence dhd expression. Nonetheless, H3K9me3 was 
always maintained at the $d h d$-distal portion of the domain. We therefore cannot exclude that this mark represses $d h d$ neighbors.

An intriguing question is then how this heterochromatin mini-domain is formed. We found that a transgene containing the full mini-domain sequence is unable to restore $\mathrm{H} 3 \mathrm{~K} 27 \mathrm{me} 3$ or $\mathrm{H} 3 \mathrm{~K} 9 \mathrm{me}$, suggesting that genomic location of this domain is a critical determinant of $d h d$ heterochromatin. From this perspective, the border elements may act as weak PREs, as described in other contexts [64]. Interestingly, we also showed that H3K9me3 can be partially maintained at the distal part of the domain in $d h d^{J 5}$ mutants while H3K27me3 is completely lost. This indicates that these marks are not necessarily inter-dependent at this locus, and $\mathrm{H} 3 \mathrm{~K} 9 \mathrm{me} 3$ may benefit from additional mechanisms ensuring its deposition. While not much is known on highly localized euchromatic deposition of H3K9me3, Smolko and colleagues suggested that Setdb1-dependent accumulation of $\mathrm{H} 3 \mathrm{~K} 9 \mathrm{me} 3$ at certain target genes is dependent on the RNA-binding protein Sxl [45]. At the dhd domain, different mechanisms could thus ensure H3K27me3 and H3K9me deposition, both of which would depend on the endogenous genomic location.

Along these lines, another recent study reported the existence of H3K27me3 microdomains (typically 2-8 nucleosomes wide) that depend on 3D contacts with larger H3K27me3 domains, mediated, in particular, by BEAF-32 and CP190 [59]. The $d$ d d mini-domain is wider and much more strongly enriched in H3K27me3 than typical micro-domains. Nonetheless, our data is consistent with a model whereby H3K27me 3 could be deposited via such looping interactions. First, BEAF-32 and CP190 are indeed found at the border elements of the $d h d$ mini-domain. Second, this mini-domain does not feature internal PREs and border elements are only weakly if at all bound by Polycomb proteins, arguing against an autonomous recruitment of E(z). Finally, a deletion of the BEAF-32/CP190-bearing regulatory element in the $d h d^{55}$ mutant, or its displacement to an ectopic genomic location in rescue transgenes both abrogate H3K27me3 deposition. Consistent with such a model, data from Heurteau et al. show a modest reduction of H3K27me3 enrichment at the $d$ hd mini-domain upon BEAF-32 depletion. Of note, BEAF-32 was also previously shown to facilitate $\mathrm{H} 3 \mathrm{~K} 9 \mathrm{me} 3$ deposition at sites featuring multiple instances of the CGATA motif, analogous to those found at the $d h d$ promoter [65]. Other studies found that ATCGAT motifs recognized by BEAF-32, also found at the $d h d$ promoter, are more broadly enriched at the promoters of Lid-activated genes [66], which is the case of $d h d$. Thus, it is possible that a BEAF-32-mediated looping mechanism is responsible for $\mathrm{H} 3 \mathrm{~K} 27 \mathrm{me} 3$ enrichment at the $d h d$ mini-domain. However, our results also show that this mark is not strictly required to repress nor to activate $d$ dhd in adults, and that Lid, Sin3A, Snrl and Mod(mdg4) activate dhd independently of it.

Scrutiny of $d h d$ regulation further uncovered how its four regulators have convergent yet distinct roles. This was particularly intriguing for Lid and Sin $3 \mathrm{~A}$, which can be found in a corepressor complex [20], at odds with their positive impact on $d h d$. Indeed, their dual depletion in cultured cells causes the misregulation of hundreds of genes [19]. Interestingly, in that study, only 55 out of 849 affected genes were similarly impacted by individual and dual knockdowns, indicating that Lid and Sin3A functionally cooperate only at a minor subset of their common targets. This seems to be the case at the $d h d$ locus, where individual KD of these factors caused an equally catastrophic collapse of transcriptional activity, suggesting a cooperative activity. Yet, Lid, but not Sin $3 \mathrm{~A}$, acted as a negative regulator of H3K27me3 at the $d h d$ locus, revealing at least partially independent functions. In contrast, Sin 3A, but not Lid, controlled the stability of regulatory elements associated with this $\mathrm{H} 3 \mathrm{~K} 27 \mathrm{me} 3$, not only at the $d h d$ domain but also genome-wide.

Our results further show a critical role for $\operatorname{Mod}(\operatorname{mdg} 4)$ as a transcriptional activator. In cell lines, ChIP-seq experiments specifically mapping the insulating $\operatorname{Mod}(\operatorname{mdg} 4) 67.2$ isoform or 
total Mod(mdg4) showed that additional isoforms are recruited to DNA [24]. Isoforms other than the 67.2 were found in particular at gene promoters in ovaries and female heads [4]. Such is the case at the $d h d$ promoter, where total Mod(mdg4) is found but not the 67.2 isoform (S2B Fig). Non-insulating roles of Mod(mdg4) were previously discussed in the context of the Polycomb-repressed Bithorax complex where the close binding of $\operatorname{Mod}(\operatorname{mgg} 4)$ to $A b d-B$ transcription start sites suggested a role in transcription activation [26]. While we cannot rule out indirect effects, these observations argue that an activating isoform of Mod(mdg4) operates directly at the $d h d$ promoter. In agreement, $\operatorname{Mod}(\operatorname{mdg} 4)$ appears to be essential to activate $d h d$ within its $\mathrm{H} 3 \mathrm{~K} 27 \mathrm{me} 3$ mini-domain, seemingly by stabilizing the $d h d$ promoter regulatory element, although its function is equally essential in the absence of heterochromatin marks in the dhd transgenic rescue construct.

The Snr1-containing Brahma complex is required for activation of target genes in Drosophila in vivo, notably during immune responses [60] and tissue regeneration [56]. In ovaries, while Snr1 has a global impact on nuclear integrity and architecture, previous immunostaining experiments interestingly showed that this factor is only expressed during a restricted time in early oogenesis [22]. This underlines the fact that $d h d$ may be dynamically regulated during oogenesis, with different regulatory components intervening at particular times. Considering that Snrl KD causes a disruption of the $d h d$ promoter-proximal regulatory element associated with $\mathrm{H} 3 \mathrm{~K} 27 \mathrm{me} 3$, this would suggest that its associated DNA-binding transcription factors also intervene during a restricted time in oogenesis. A precise dissection of the timing of $d h d$ transcription, and determining whether these factors target $d h d$ directly and simultaneously, would be essential to understand the cascade of events leading to its massive expression.

The case of $d h d$ indeed illustrates the complexity of understanding the chromatin landscape at cell type-specific genes, when the starting material is a complex tissue. In this context, the Cut\&Run analysis implemented in our study allowed us to reveal the co-occupancy of H3K27me3 nucleosomes and associated transcription factors. While this approach cannot identify the cell of origin of each individual DNA molecule, it can be used to make important deductions on the combinatorial co-occupancy on DNA of different chromatin components. This approach joins other recent methods comparable in their principle, namely the DNA methyl-transferase single-molecule footprinting (dSMF) method [67] and the low-salt antibody-targeted tagmentation (CUTAC) approach [68]. Together with single-cell methodologies, these approaches hold the potential to begin uncovering complex epigenomic regulation processes, such as that of $d h d$, that were until recently inaccessible.

\section{Materials \& methods}

\section{Drosophila strains}

Flies were raised at $25^{\circ} \mathrm{C}$ on standard medium. The following stocks were obtained from the Bloomington Drosophila Stock Center (simplified genotypes are given): P\{TRiP.HMS00849\} attP2 (mod(mdg4) shRNA; \#33907), P\{TRiP. HMS00363\}attP2 (Snr1 shRNA; \#32372), P\{TRiP. GL00612\}attP40 (lid shRNA; \#36652), P\{TRiP.GLV21071\}attP2 (lid shRNA; \#35706), P\{TRiP. HMS00359\}attP2 (Sin3a shRNA; \#32368), P\{TRiP.HMS00066\}attP2 (E(z) shRNA; \#33659), P $\{y[+t 7.7]=$ CaryP $\}$ attP2 (Control line for TRiP RNAi lines; \#B36303), P\{otu-GAL4::VP16.R $\} 1$; P\{GAL4-nos.NGT\}40; P\{GAL4::VP16-nos.UTR $\} M V D 1$ (Maternal Triple Driver or "MTDGal4"; \#31777), P\{GAL4::VP16-nos.UTR\}MVD1 ("nos-Gal4"; \#4937). Other stocks are: $w^{1118}$, $D f(1) J 5 / F M 7 c$ [7], P[Mst35Ba-EGFP] [27], $p W 8-d h d^{W T}$ [9]. TRiP lines target all predicted isoforms of their respective target genes. "Control" in shRNA experiments refers to the offspring of the control line for TRiP lines crossed with the MTD-Gal4 line. 
For the $p W 8-d h d^{\triangle D R E}$ mutant, two fragments were amplified by PCR from $w^{1118}$ genomic DNA using the primers $\triangle \mathrm{DRE}-1$-for/ $\triangle \mathrm{DRE}-1$-rev and $\triangle \mathrm{DRE}-2$-for/ $\triangle \mathrm{DRE}-2$-rev (S1 Table). PCR products were assembled and cloned into the $p W 8-d h d^{W T}$ vector [9] previously digested by KpnI and BamHI using the NEBuilder HiFi DNA Assembly Cloning Kit (NEB, \#E5520S). $d h d^{\Delta \mathrm{DRE}}$ transgene was integrated in the PBac\{attP-3B\}VK00031 platform (62E1) using PhiC31-mediated transformation and flies were generated by The Best Gene (TheBestGene.com).

\section{Germline knock-down and fertility tests}

To obtain $K D$ females, virgin shRNA transgenic females were mass crossed with transgenic Gal4 males at $25^{\circ} \mathrm{C}$ and females of the desired genotype were recovered in the $\mathrm{F} 1$ progeny. All RNAi experiments were carried at $25^{\circ} \mathrm{C}$. To measure fertility, virgin females of different genotypes were mated to males in a 1:1 ratio and placed for 2 days at $25^{\circ} \mathrm{C}$. They were then transferred to a new vial and allowed to lay eggs for 24 hours. Embryos were counted and then let to develop for at least 36 hours at $25^{\circ} \mathrm{C}$. Unhatched embryos were counted to determine hatching rates.

\section{Gene expression analysis by RT-QPCR}

Total RNA was extracted from ovaries of 3-day-old females using the NucleoSpin RNA isolation kit (Macherey-Nagel), following the instructions of the manufacturer. $1 \mu \mathrm{g}$ of total RNA was reverse transcribed using the SuperScript II Reverse Trancriptase kit (Invitrogen) with oligo (dT) primers. RT-qPCR reactions were performed in duplicates as described previously [5]. Primer sets used are provided in S1 Table. Statistical tests were performed using GraphPad Prism version 9.2.0 for Mac OS (GraphPad Software).

\section{Immunofluorescence and imaging}

Early (0-30 min) embryos laid by females of the indicated genotypes were collected on agar plates. Embryos were dechorionated in bleach, fixed in a 1:1 heptane:methanol mixture and stored at $-20^{\circ} \mathrm{C}$. Embryos were washed three times (10 min each) with PBS $0.1 \%$, Triton X-100 (PBS-T) and then incubated with primary antibodies in the same buffer on a wheel overnight at $4^{\circ} \mathrm{C}$. They were then washed three times $(20 \mathrm{~min}$ each) with PBS-T. Incubations with secondary antibodies were performed identically. Embryos were mounted in DAKO mounting medium containing DAPI.

Ovaries were dissected in PBS-T and fixed at room temperature in $4 \%$ formaldehyde in PBS for 25 minutes. Immunofluorescence was performed as for embryos. Ovaries were then mounted as described above.

Antibodies used are provided in S2 Table. Images were acquired on an LSM 800 confocal microscope (Carl Zeiss). Images were processed with Zen imaging software (Carl Zeiss) and ImageJ software.

\section{Western blotting}

Ovaries from 30 females were collected and homogenized in lysis buffer (20mM Hepes pH7.9, $100 \mathrm{mM} \mathrm{KCl}, 0.1 \mathrm{mM}$ EDTA, $0.1 \mathrm{mM}$ EGTA, 5\% Glycerol, 0.05\% Igepal and protease inhibitors (Roche)). Protein extracts were cleared by centrifugation and purified with Pierce GST Spin Purification Kit (ThermoFisher Scientific, \#16106). Western analysis was performed using standard procedures and used antibodies and concentrations are presented in S2 Table. 


\section{Ovarian RNA sequencing and analysis}

Samples were processed as previously described [5].

Sequencing was completed on two biological replicates of the following genotypes: $\bmod (m d g 4) \mathrm{KD}(M T D-G a l 4>$ shRNA $\bmod (\operatorname{mdg} 4))$, i.e $P\{w[+m C]=$ otu-GAL4::VP16.R $\} 1, w\left[^{*}\right] / y[1] s c\left[^{*}\right] v[1] ; P\{w[+m C]=$ GAL4-nos.NGT\}40/+; $P\{w[+m C]=$ GAL4::VP16-nos.UTR $\}$ CG6325[MVD1] $/ \mathrm{P}\{\mathrm{y}[+\mathrm{t} 7.7] \mathrm{v}[+\mathrm{t} 1.8]=$ TRiP. HMS00849 $\}$ attP2

Snr1 KD (MTD-Gal4>shRNA Snr1), i.e

$\mathrm{P}\{\mathrm{w}[+\mathrm{mC}]=$ otu-GAL4::VP16.R $\} 1, \mathrm{w}\left[^{*}\right] / \mathrm{y}[1] \mathrm{sc}\left[{ }^{*}\right] \mathrm{v}[1] ; \mathrm{P}\{\mathrm{w}[+\mathrm{mC}]=$ GAL4-nos.NGT $\} 40 /+$; $\mathrm{P}\{\mathrm{w}[+\mathrm{mC}]=\mathrm{GAL} 4:$ VP16-nos.UTR $\}$ CG6325[MVD1] $/ \mathrm{P}\{\mathrm{y}[+\mathrm{t} 7.7] \mathrm{v}[+\mathrm{t} 1.8]=\mathrm{TRiP}$. HMS00363\}attP2

\section{Chromatin profiling by CUT\&RUN}

Cut\&Run in Drosophila tissues was previously described [37]. Briefly, ovaries from 3-day-old flies were dissected in Wash+ Buffer (20 mM HEPES pH 7.5, $150 \mathrm{mM} \mathrm{NaCl}, 0.9 \mathrm{mM}$ spermidine, $0.1 \%$ BSA with cOmplete protease inhibitor, Roche) and were bound to BioMag Plus Concanavalin-A-conjugated magnetic beads (ConA beads, Polysciences, Inc). Tissues were then permeabilized for 10min in dbe+ Buffer $(20 \mathrm{mM}$ HEPES pH 7.5, $150 \mathrm{mM} \mathrm{NaCl}, 0.9 \mathrm{mM}$ spermidine, $2 \mathrm{mM}$ EDTA, 0.1\% BSA, 0.05\% digitonin and protease inhibitors). Samples were then incubated with gentle rocking overnight at $4^{\circ} \mathrm{C}$ with primary antibody solution in dbe + buffer (see S2 Table for antibody concentrations). Protein A fused to micrococcal nuclease (p-AMNase) was added in dbe+ buffer and samples were incubated with rotation at room temperature for 1 hour. Cleavage was done in WashCa+ buffer $(20 \mathrm{mM}$ HEPES pH 7.5, $150 \mathrm{mM}$ $\mathrm{NaCl}, 0.9 \mathrm{mM}$ spermidine, $0.1 \% \mathrm{BSA}, 2 \mathrm{mM} \mathrm{CaCl} 2$ with and protease inhibitors) at $0^{\circ}$ for 30 minutes. Digestion was stopped with addition of 2XSTOP Buffer $(200 \mathrm{mM} \mathrm{NaCl}, 20 \mathrm{mM}$ EDTA, $4 \mathrm{mM}$ EGTA, $62.5 \mu \mathrm{g} / \mathrm{mL}$ RNaseA). Samples were incubated at $37^{\circ} \mathrm{C}$ for $30 \mathrm{~min}$ to digest RNA and release DNA fragments. Cleaved DNA was then recovered with Ampure XP beads (Beckman Coulter) immediately after protease treatment. Antibodies used for CUT\&RUN are listed in S2 Table. Retrieved DNA was used either for qPCR or for library preparation followed by deep sequencing. Sequencing libraries for each sample were synthesized using Diagenode MicroPlex Library Preparation kit according to supplier recommendations (version 2.02.15) and were sequenced on Illumina Hiseq 4000 sequencer as Paired-End 100 base reads following Illumina's instructions (GenomEast platform, IGBM, Strasbourg, France). Image analysis and base calling were performed using RTA 2.7.7 and bcl2fastq 2.17.1.14. Adapter dimer reads were removed using DimerRemover.

\section{Cut\&Rut-qPCR}

$0,1 \mathrm{ng}$ of retrieved DNA in Cut\&Run were used as template in a real time quantitative PCR assay using SYBR Premix Ex Taq II (Tli RNaseH Plus) (Takara). All qPCR reactions were performed in duplicates using Bio-Rad CFX-96 Connect system with the following conditions: $95^{\circ} \mathrm{C}$ for 30 s followed by 40 cycles of denaturation at $95^{\circ} \mathrm{C}$ for $5 \mathrm{~s}$ and annealing and extension at $59^{\circ} \mathrm{C}$ for 30 s. As a normalization control, we processed ovary samples from each studied genotype as for Cut\&Run, except the antibody and pA-MNase incubation steps were omitted and instead we incubated tissue with $10 \mathrm{U}$ of Micrococcal Nuclease for $30 \mathrm{~min}$ at $37^{\circ} \mathrm{C}$ (ThermoFisher Scientific, \#88216). Fold change in histone mark enrichment was determined relative to this whole MNase control and relative to the Sas10 gene, which was depleted in the histone marks tested in this study. Primer sets used are provided in S1 Table. 


\section{Sequencing data processing}

Paired-end reads were mapped to the release 6 of the D. melanogaster genome using Bowtie2 (v. 2.4.2). To compare samples with identical readcount for genome coverage quantifications, we employed Downsample SAM/BAM (Galaxy Version 2.18.2.1). To obtain short fragment datasets for DNA regulatory element identification, peak calling and visualization, we selected fragments shorter than $120 \mathrm{bp}$ from SAM files. These were typically a small minority of all fragments, as our Cut\&Run datasets were largely dominated by nucleosome-sized fragments (150-250bp). We therefore could separately analyze modification-bearing nucleosome coverage (for which the complete Cut\&Run dataset, "All fragments", was a good approximation) or putative regulatory element coverage ( $<120 \mathrm{bp}$ fragments). For genomic track visualization, we used bamCoverage from deepTools 2.0 (Galaxy Version 3.3.2.0.0) to calculate read coverage per 25bp bin for all fragments or 10bp bin for short fragments, with paired-end extension. Peak calling was done on sorted short fragments ( $<120$ bp) with MACS2 (v. 2.1.1.20160309) with the following parameters:-nomodel,-p-value $=0.0001,-$ keep-dup $=$ all and the rest by default. To establish a high-confidence short fragment peak list we retained peaks that were present in biological replicates from the control genotype. Genome browser views screenshots were produced with the IGV software, for Cut\&Run we used a 25bp bin for all fragments and a 10bp bin for short fragments ( $<120 \mathrm{bp}$ ). For the midpoint-plot of fragment sizes around short fragment peaks, the length of each fragment was plotted as a function of the distance from the fragment midpoint to the summit of the peak identified by MACS2. For signal quantification at the $d h d$ locus, normalized read counts were counted within the domain (Coordinates $[5,312,54-5,317,465])$ or within border element peaks (5' element: $[5,312,120-$ 5,312,211], 3' element: [5,317,300-5,317,447])

Heatmaps were generated with RStudio (RStudio Team (2016). RStudio: Integrated Development for R. RStudio, Inc., Boston, MA URL) and the packages 'gplots' (v.3.1.1) and 'plyr' (Wickham, 2011).

\section{Motif scanning}

Motif scanning on the $p W 8-d h d^{W T}$ transgene sequence was done with FIMO (v. 5.3.3) [69] using the flyreg v. 2 motif database with default parameters.

\section{Supporting information}

S1 Fig. $\bmod (\boldsymbol{m d g} 4) \mathrm{KD}$ and $\operatorname{Snr} 1 \mathrm{KD}$ downregulate $\boldsymbol{d h d}$. A-mod(mdg4) KD and Snr1 KD are efficient in the female germline. Left: RT-qPCR quantification of mod(mdg4) (top) or Snr1 (bottom) mRNA levels in Control and KD ovaries (normalized to rp49 and relative to expression in Control ovaries). Data from biological duplicates analyzed in technical duplicates are presented as mean \pm SEM. Right: Quantification of $\bmod (\operatorname{mdg} 4)$ (top) and Snrl(bottom) counts in RNA-seq data from Fig 1B. Both duplicates are shown. B-lid, Sin3a, Snr1 and mod(mdg4) KDs downregulate $d h d$ but do not significantly affect its neighboring genes. Quantification of counts in RNA-seq data for $d h d$ and its neighboring genes in Control, lid, Sin3a, Snr1 and mod (mdg4) KD show that low-expressing genes in the $d h d$ region are not or only modestly impacted by the KDs. Both duplicates are shown. C-Limited overlap in the effects of mod ( $m d g 4)$ and Snr1 KDs. Hierarchical clustering of sample distance heatmap of RNA-seq samples. D-Principal component analysis for RNA-seq samples. E- lid, Sin3a, $\bmod (\operatorname{mdg} 4)$ and Snr1 KD severely downregulate $d h d$ expression. RT-qPCR quantification of $d h d$ mRNA levels in ovaries of indicated genotypes (normalized to $r p 49$ and relative to expression in Control ovaries). Two different shRNA constructs (val21 and val22) against lid were tested. Data from 
biological duplicates analyzed in technical duplicates are presented as mean \pm SEM.

(TIF)

S2 Fig. Cut\&Run is consistent with ChIP-seq data. A-Histone modification profiles at the dhd region in cultured embryonic cells and ovaries. ChIP-seq data showing the active mark H3K4me3 (yellow) [5], and the repressive marks H3K9me3 (green) [40,45] and H3K27me3 (blue) (accession number GSE145282, [50]). B-Short fragment peaks align with known regulatory elements. Genome browser views of the bithorax complex (BX-C) (left) and the $d h d$ region (right). Display of H3K27me3 Cut\&Run (from Control ovaries, all fragments and $<120$ bp fragments), ATAC-seq (from S2 cells, [35]), CP190 ChIP-seq (from Kc cells, [38]), $\operatorname{Mod}(\mathrm{mdg} 4)$ (all isoforms) and Mod(mdg4)67.2 isoform ChIP-seq (from Kc cells, [24]) Polycomb (Pc) and Polyhomeotic (Ph) ChIP-seq (from S2 cells, [37]). Cut\&Run short fragments largely overlap with peaks from the other tracks displayed.

S3 Fig. Cut\&Run maps H3K9me3 in ovaries. A- Cut\&Run shows the expected enrichment of $\mathrm{H} 3 \mathrm{~K} 9 \mathrm{me} 3$ at pericentromeric heterochromatin. Genome browser views of H3K9me3 ChIPseq [45] and Cut\&Run signal in all chromosomes. B- Cut\&Run detects a previously identified H3K9me3 peak over a testis-specific TSS [45]. Genome browser view of phf7 and neighboring genes. Blue arrow indicates testis-specific TSS and magenta arrow indicates ovary-specific TSS.

(TIF)

S4 Fig. Whole-ovary experiments yield signal from both somatic follicle cells and germline cells. A-E $(z)$ KD does not severely affect $d h d$ expression. RT-qPCR quantification of $d h d$ mRNA levels in Control and $E(z) \mathrm{KD}$ ovaries (normalized to $r p 49$ and relative to expression in Control ovaries). Data from biological duplicates analyzed in technical duplicates are presented as mean \pm SEM. B- $E(z) \mathrm{KD}$ and Snr1 KD affect H3K27me3 levels in nurse cells. Confocal images of representative egg chambers in Control, $E(z) \mathrm{KD}$, lid KD, Sin3a KD, $\bmod (\operatorname{mdg} 4)$ $\mathrm{KD}$ and Snr1 KD. In control ovaries, H3K27me3 staining marks somatic follicle cell nuclei, the karyosome and germline nurse cell nuclei. In $E(z) \mathrm{KD}$ ovaries the karyosome and nurse cells loose staining of the histone mark but follicle cells are marked normally. No notable change is observed in lid KD, Sin $3 a$ KD or mod(mdg4) KD while in Snr1 KD nurse cells staining is less intense. Scale bar $10 \mu \mathrm{m}$. C- Cut\&Run in whole ovaries captures signal from both somatic and germline cells. Genome browser views of $\mathrm{H} 3 \mathrm{~K} 27 \mathrm{me} 3 \mathrm{Cut} \&$ Run signal in Control and $E(z) \mathrm{KD}$ ovaries and H3K27me3 ChIP-seq from FACS sorted nurse cells and somatic follicle cells [50]. Upper panels show representative loci enriched for the mark solely in nurse cells (germline) and absent in $E(z) \mathrm{KD}$ ovaries. Lower panels show H3K27me3 domains where the signal comes almost exclusively from follicle cells and is not significantly affected in the germline $E$ (z) KD.

S5 Fig. Cut\&Run is reproducible among replicates. A-H3K27me3 Cut\&Run signal at the dhd locus from Control and KD ovaries. Left: Dotplot showing normalized read counts of H3K27me3 Cut\&Run at the dhd domain from independent biological triplicates of the indicated genotypes (duplicates for $E(z) \mathrm{KD}$ ). B-Cut\&Run qPCR yields reproducible data among replicates. Biological replicates from H3K27me3 and H3K9me3 Cut\&Run-qPCR in Control and $\mathrm{KD}$ ovaries shown in Fig 3E. The Sas10 gene was used as negative control and Ubx and CG12239 as positive controls for H3K27me3 and H3K9me3 respectively. Fold enrichment was calculated relative to Sas10. Error bars show technical variability. C-Sin $3 a \mathrm{KD}$, Snr1 KD and $\bmod (m d g 4) \mathrm{KD}$ affect the stability of the H3K27me3-associated regulatory elements at the $d h d$ 
mini-domain. Dotplot showing normalized read counts of H3K27me3 Cut\&Run $<120 \mathrm{bp}$ fragments at $d h d$ regulatory elements from independent biological triplicates of the indicated genotypes (duplicates for $E(z) \mathrm{KD}$ ). 5' and 3' border elements are plotted separately.

S6 Fig. Dref and Beaf-32 are found at $\boldsymbol{d} \boldsymbol{h} \boldsymbol{d}$ regulatory elements. Genome browser views of ovarian H3K27me3 Cut\&Run (all fragments and $<120 \mathrm{bp}$ fragments) and Dref and Beaf-32 ChIP-seq (from Kc cells, [38]). $<120 \mathrm{bp}$ fragment peaks at the $d$ hd domain borders align with DREF and Beaf-32 peaks.

S7 Fig. Cut\&Run qPCR generates reproducible data from transgenes. Biological replicates of H3K27me3 and H3K9me3 Cut\&Run-qPCR in Control, $d h d^{J 5}$, $d h d^{J 5} ; ; p W 8-d h d^{W T}$ and $d h d^{J 5} ; p$ p $8-d h d^{F D}$ ovaries shown in Fig 5D. Fold enrichment was calculated relative to Sas10. Error bars show technical variability.

S8 Fig. In lid KD, Sin3a KD, mod(mdg4) KD and Snr1 KD rescue flies, H3K27me3 is absent from the $\boldsymbol{d} \boldsymbol{h} \boldsymbol{d}$ rescue transgene. $\mathrm{A}-\operatorname{lid} \mathrm{KD}, \operatorname{Sin} 3 a \mathrm{KD}, \bmod (m d g 4) \mathrm{KD}$ and $\operatorname{Snr} 1 \mathrm{KD}$ are efficient in the female germline of rescue flies. From left to right: RT-qPCR quantification of lid, $\operatorname{Sin} 3 a, \bmod (m d g 4)$ and Snr 1 mRNA levels in ovaries of the indicated genotypes (normalized to $r p 49$ and relative to expression in $w^{1118}$ ovaries). Data from biological duplicates analyzed in technical duplicates are presented as mean \pm SEM. $\mathrm{B}$-The $d$ hd rescue transgene does not restore H3K27me3 in KD flies. Biological replicates of H3K27me3 Cut\&Run-qPCR in the indicated genotypes shown in Fig 6C. The Sas10 gene was used as negative control and Ubx as positive control. Fold enrichment was calculated relative to Sas10. Error bars show technical variability.

(TIF)

S1 Table. List of primers used in this paper.

S2 Table. List of antibodies used in this paper.

(PDF)

\section{Acknowledgments}

We thank Dr. Kami Ahmad for sharing reagents and advice on Cut\&Run. We are grateful to the TRiP projects and Bloomington Drosophila Stock Center for fly stocks. We acknowledge the contribution of SFR Biosciences (UAR3444/CNRS, US8/Inserm, ENS de Lyon, UCBL) facilities PLATIM and Arthro-tools. Sequencing was performed by the GenomEast platform, a member of the 'France Génomique' consortium. We would like to thank Raphaëlle Dubruille and Francesca Palladino for critical reading of the manuscript. We also warmly thank them, as well as Lucas Waltzer and all members of the Loppin lab for their input throughout this project.

\section{Author Contributions}

Conceptualization: Daniela Torres-Campana, Benjamin Loppin, Guillermo A. Orsi.

Data curation: Daniela Torres-Campana, Gérard Benoit, Guillermo A. Orsi.

Formal analysis: Daniela Torres-Campana, Gérard Benoit, Guillermo A. Orsi. 
Funding acquisition: Benjamin Loppin, Guillermo A. Orsi.

Investigation: Daniela Torres-Campana, Béatrice Horard, Sandrine Denaud, Benjamin Loppin, Guillermo A. Orsi.

Methodology: Daniela Torres-Campana, Guillermo A. Orsi.

Supervision: Béatrice Horard, Benjamin Loppin, Guillermo A. Orsi.

Validation: Daniela Torres-Campana, Guillermo A. Orsi.

Writing - original draft: Daniela Torres-Campana, Guillermo A. Orsi.

Writing - review \& editing: Daniela Torres-Campana, Béatrice Horard, Gérard Benoit, Benjamin Loppin, Guillermo A. Orsi.

\section{References}

1. Bannister AJ, Kouzarides T. Regulation of chromatin by histone modifications. Cell Res. 2011;21:381395. https://doi.org/10.1038/cr.2011.22 PMID: 21321607

2. Hammond CM, Strømme CB, Huang H, Patel DJ, Groth A. Histone chaperone networks shaping chromatin function. Nat Rev Mol Cell Biol. 2017; 18: 141-158. https://doi.org/10.1038/nrm.2016.159 PMID: 28053344

3. Tyagi M, Imam N, Verma K, Patel AK. Chromatin remodelers: We are the drivers!! Nucleus. 2016; 7: 388-404. https://doi.org/10.1080/19491034.2016.1211217 PMID: 27429206

4. Melnikova LS, Georgiev PG, Golovnin AK. The Functions and Mechanisms of Action of Insulators in the Genomes of Higher Eukaryotes. Acta Naturae. 2020; 12: 15-33. https://doi.org/10.32607/actanaturae. 11144 PMID: 33456975

5. Torres-Campana D, Kimura S, Orsi GA, Horard B, Benoit G, Loppin B. The Lid/KDM5 histone demethylase complex activates a critical effector of the oocyte-to-zygote transition. Bosco G, editor. PLoS Genet. 2020; 16: e1008543. https://doi.org/10.1371/journal.pgen.1008543 PMID: 32134927

6. Spradling A. The development of Drosophila melanogaster. 1993.

7. Salz HK, Flickinger TW, Mittendorf E, Pellicena-Palle A, Petschek JP, Albrecht EB. The Drosophila maternal effect locus deadhead encodes a thioredoxin homolog required for female meiosis and early embryonic development. Genetics. 1994; 136: 1075-1086. https://doi.org/10.1093/genetics/136.3. 1075 PMID: 7516301

8. Svensson MJ, Chen JD, Pirrotta V, Larsson J. The ThioredoxinT and deadhead gene pair encode testis- and ovary-specific thioredoxins in Drosophila melanogaster. Chromosoma. 2003; 112: 133-143. https://doi.org/10.1007/s00412-003-0253-5 PMID: 14579129

9. Tirmarche S, Kimura S, Dubruille R, Horard B, Loppin B. Unlocking sperm chromatin at fertilization requires a dedicated egg thioredoxin in Drosophila. Nat Commun. 2016; 7: 13539. https://doi.org/10 1038/ncomms13539 PMID: 27876811

10. Petrova B, Liu K, Tian C, Kitaoka M, Freinkman E, Yang J, et al. Dynamic redox balance directs the oocyte-to-embryo transition via developmentally controlled reactive cysteine changes. Proc Natl Acad Sci USA. 2018; 115: E7978-E7986. https://doi.org/10.1073/pnas.1807918115 PMID: 30082411

11. Freier R, Aragón E, Bagiński B, Pluta R, Martin-Malpartida $P$, Ruiz L, et al. Structures of the germlinespecific Deadhead and thioredoxin T proteins from Drosophila melanogaster reveal unique features among thioredoxins. IUCrJ. 2021; 8: 281-294. https://doi.org/10.1107/S2052252521000221 PMID: 33708404

12. Emelyanov AV, Fyodorov DV. Thioredoxin-dependent disulfide bond reduction is required for protamine eviction from sperm chromatin. Genes Dev. 2016; 30: 2651-2656. https://doi.org/10.1101/gad.290916. 116 PMID: 28031247

13. Dingwall AK, Beek SJ, McCallum CM, Tamkun JW, Kalpana GV, Goff SP, et al. The Drosophila snr1 and brm proteins are related to yeast SWI/SNF proteins and are components of a large protein complex. MBoC. 1995; 6: 777-791. https://doi.org/10.1091/mbc.6.7.777 PMID: 7579694

14. Gerasimova TI, Gdula DA, Gerasimov DV, Simonova O, Corces VG. A drosophila protein that imparts directionality on a chromatin insulator is an enhancer of position-effect variegation. Cell. 1995; 82: 587597. https://doi.org/10.1016/0092-8674(95)90031-4 PMID: 7664338

15. Skene PJ, Henikoff $S$. An efficient targeted nuclease strategy for high-resolution mapping of DNA binding sites. eLife. 2017; 6: e21856. https://doi.org/10.7554/eLife.21856 PMID: 28079019 
16. Hirose F, Yamaguchi M, Handa H, Inomata Y, Matsukage A. Novel 8-base pair sequence (Drosophila DNA replication-related element) and specific binding factor involved in the expression of Drosophila genes for DNA polymerase alpha and proliferating cell nuclear antigen. J Biol Chem. 1993; 268: 20922099. PMID: 8093616

17. Spain MM, Caruso JA, Swaminathan A, Pile LA. Drosophila SIN3 isoforms interact with distinct proteins and have unique biological functions. J Biol Chem. 2010; 285: 27457-27467. https://doi.org/10.1074/ jbc.M110.130245 PMID: 20566628

18. Das TK, Sangodkar J, Negre N, Narla G, Cagan RL. Sin3a acts through a multi-gene module to regulate invasion in Drosophila and human tumors. Oncogene. 2013; 32: 3184-3197. https://doi.org/10.1038/ onc.2012.326 PMID: 22890320

19. Gajan A, Barnes VL, Liu M, Saha N, Pile LA. The histone demethylase dKDM5/LID interacts with the SIN3 histone deacetylase complex and shares functional similarities with SIN3. Epigenetics \& Chromatin. 2016; 9: 4. https://doi.org/10.1186/s13072-016-0053-9 PMID: 26848313

20. Moshkin YM, Kan TW, Goodfellow H, Bezstarosti K, Maeda RK, Pilyugin M, et al. Histone Chaperones ASF1 and NAP1 Differentially Modulate Removal of Active Histone Marks by LID-RPD3 Complexes during NOTCH Silencing. Molecular Cell. 2009; 35: 782-793. https://doi.org/10.1016/j.molcel.2009.07. 020 PMID: 19782028

21. Ni J-Q, Zhou R, Czech B, Liu L-P, Holderbaum L, Yang-Zhou D, et al. A genome-scale shRNA resource for transgenic RNAi in Drosophila. Nat Methods. 2011; 8: 405-407. https://doi.org/10.1038/nmeth.1592 PMID: 21460824

22. Zraly CB, Marenda DR, Nanchal R, Cavalli G, Muchardt C, Dingwall AK. SNR1 is an essential subunit in a subset of Drosophila brm complexes, targeting specific functions during development. Dev Biol. 2003; 253: 291-308. https://doi.org/10.1016/s0012-1606(02)00011-8 PMID: 12645932

23. Dorn R, Reuter $\mathrm{G}$, Loewendorf $A$. Transgene analysis proves mRNA trans-splicing at the complex mod (mdg4) locus in Drosophila. Proc Natl Acad Sci U S A. 2001; 98: 9724-9729. https://doi.org/10.1073/ pnas.151268698 PMID: 11493677

24. Van Bortle K, Ramos E, Takenaka N, Yang J, Wahi JE, Corces VG. Drosophila CTCF tandemly aligns with other insulator proteins at the borders of H3K27me3 domains. Genome Res. 2012; 22: 21762187. https://doi.org/10.1101/gr.136788.111 PMID: 22722341

25. Büchner K, Roth P, Schotta G, Krauss V, Saumweber H, Reuter G, et al. Genetic and Molecular Complexity of the Position Effect Variegation Modifier mod(mdg4) in Drosophila. Genetics. 2000; 155: 141157. https://doi.org/10.1093/genetics/155.1.141 PMID: 10790390

26. Savitsky M, Kim M, Kravchuk O, Schwartz YB. Distinct Roles of Chromatin Insulator Proteins in Control of the Drosophila Bithorax Complex. Genetics. 2016; 202: 601-617. https://doi.org/10.1534/genetics. 115.179309 PMID: 26715665

27. Manier MK, Belote JM, Berben KS, Novikov D, Stuart WT, Pitnick S. Resolving mechanisms of competitive fertilization success in Drosophila melanogaster. Science. 2010; 328: 354-357. https://doi.org/10. 1126/science.1187096 PMID: 20299550

28. Henikoff JG, Belsky JA, Krassovsky K, MacAlpine DM, Henikoff S. Epigenome characterization at single base-pair resolution. Proceedings of the National Academy of Sciences. 2011; 108: 18318-18323. https://doi.org/10.1073/pnas.1110731108 PMID: 22025700

29. Kasinathan S, Orsi GA, Zentner GE, Ahmad K, Henikoff S. High-resolution mapping of transcription factor binding sites on native chromatin. Nat Methods. 2014; 11: 203-209. https://doi.org/10.1038/nmeth. 2766 PMID: 24336359

30. Orsi GA, Kasinathan S, Hughes KT, Saminadin-Peter S, Henikoff S, Ahmad K. High-resolution mapping defines the cooperative architecture of Polycomb response elements. Genome Research. 2014; 24 809-820. https://doi.org/10.1101/gr.163642.113 PMID: 24668908

31. Ramachandran S, Ahmad K, Henikoff S. Transcription and Remodeling Produce Asymmetrically Unwrapped Nucleosomal Intermediates. Molecular Cell. 2017; 68: 1038-1053.e4. https://doi.org/10. 1016/j.molcel.2017.11.015 PMID: 29225036

32. Cleard F, Wolle D, Taverner AM, Aoki T, Deshpande G, Andolfatto P, et al. Different Evolutionary Strategies To Conserve Chromatin Boundary Function in the Bithorax Complex. Genetics. 2017; 205: 589603. https://doi.org/10.1534/genetics.116.195586 PMID: 28007886

33. Kyrchanova O, Mogila V, Wolle D, Deshpande G, Parshikov A, Cléard F, et al. Functional Dissection of the Blocking and Bypass Activities of the Fab-8 Boundary in the Drosophila Bithorax Complex. Sánchez-Herrero E, editor. PLoS Genet. 2016; 12: e1006188. https://doi.org/10.1371/journal.pgen. 1006188 PMID: 27428541

34. Ahmad K, Spens AE. Separate Polycomb Response Elements control chromatin state and activation of the vestigial gene. Cavalli G, editor. PLoS Genet. 2019; 15: e1007877. https://doi.org/10.1371/journal. pgen.1007877 PMID: 31425502 
35. Jain SU, Rashoff AQ, Krabbenhoft SD, Hoelper D, Do TJ, Gibson TJ, et al. H3 K27M and EZHIP Impede H3K27-Methylation Spreading by Inhibiting Allosterically Stimulated PRC2. Mol Cell. 2020; 80: 726-735.e7. https://doi.org/10.1016/j.molcel.2020.09.028 PMID: 33049227

36. Minoux M, Holwerda S, Vitobello A, Kitazawa T, Kohler H, Stadler MB, et al. Gene bivalency at Polycomb domains regulates cranial neural crest positional identity. Science. 2017; 355: eaal2913. https:// doi.org/10.1126/science.aal2913 PMID: 28360266

37. Enderle D, Beisel C, Stadler MB, Gerstung M, Athri P, Paro R. Polycomb preferentially targets stalled promoters of coding and noncoding transcripts. Genome Res. 2011;21:216-226. https://doi.org/10. 1101/gr.114348.110 PMID: 21177970

38. Li L, Lyu X, Hou C, Takenaka N, Nguyen HQ, Ong C-T, et al. Widespread Rearrangement of 3D Chromatin Organization Underlies Polycomb-Mediated Stress-Induced Silencing. Molecular Cell. 2015; 58 : 216-231. https://doi.org/10.1016/j.molcel.2015.02.023 PMID: 25818644

39. Bartkuhn M, Straub T, Herold M, Herrmann M, Rathke C, Saumweber H, et al. Active promoters and insulators are marked by the centrosomal protein 190. EMBO J. 2009; 28: 877-888. https://doi.org/10. 1038/emboj.2009.34 PMID: 19229299

40. Colmenares SU, Swenson JM, Langley SA, Kennedy C, Costes SV, Karpen GH. Drosophila Histone Demethylase KDM4A Has Enzymatic and Non-enzymatic Roles in Controlling Heterochromatin Integrity. Developmental Cell. 2017; 42: 156-169.e5. https://doi.org/10.1016/j.devcel.2017.06.014 PMID: 28743002

41. Kassis JA, Kennison JA, Tamkun JW. Polycomb and Trithorax Group Genes in Drosophila. Genetics. 2017; 206: 1699-1725. https://doi.org/10.1534/genetics.115.185116 PMID: 28778878

42. Schuettengruber B, Bourbon H-M, Di Croce L, Cavalli G. Genome Regulation by Polycomb and Trithorax: 70 Years and Counting. Cell. 2017; 171: 34-57. https://doi.org/10.1016/j.cell.2017.08.002 PMID: 28938122

43. Brower-Toland B, Riddle NC, Jiang H, Huisinga KL, Elgin SCR. Multiple SET methyltransferases are required to maintain normal heterochromatin domains in the genome of Drosophila melanogaster. Genetics. 2009; 181: 1303-1319. https://doi.org/10.1534/genetics.108.100271 PMID: 19189944

44. Ninova M, Fejes Tóth $\mathrm{K}$, Aravin AA. The control of gene expression and cell identity by $\mathrm{H} 3 \mathrm{~K} 9$ trimethylation. Development. 2019; 146: dev181180. https://doi.org/10.1242/dev.181180 PMID: 31540910

45. Smolko AE, Shapiro-Kulnane L, Salz HK. The H3K9 methyltransferase SETDB1 maintains female identity in Drosophila germ cells. Nat Commun. 2018; 9: 4155. https://doi.org/10.1038/s41467-018-06697-x PMID: 30297796

46. Kadoch C, Williams RT, Calarco JP, Miller EL, Weber CM, Braun SMG, et al. Dynamics of BAF-Polycomb complex opposition on heterochromatin in normal and oncogenic states. Nat Genet. 2017; 49: 213-222. https://doi.org/10.1038/ng.3734 PMID: 27941796

47. De S, Gehred ND, Fujioka M, Chan FW, Jaynes JB, Kassis JA. Defining the Boundaries of Polycomb Domains in Drosophila. Genetics. 2020; 216: 689-700. https://doi.org/10.1534/genetics.120.303642 PMID: 32948625

48. Iovino N, Ciabrelli F, Cavalli G. PRC2 controls Drosophila oocyte cell fate by repressing cell cycle genes. Dev Cell. 2013; 26: 431-439. https://doi.org/10.1016/j.devcel.2013.06.021 PMID: 23932903

49. Phillips MD, Shearn A. Mutations in polycombeotic, a Drosophila polycomb-group gene, cause a wide range of maternal and zygotic phenotypes. Genetics. 1990; 125: 91-101. https://doi.org/10.1093/ genetics/125.1.91 PMID: 2341036

50. DeLuca SZ, Ghildiyal M, Pang L-Y, Spradling AC. Differentiating Drosophila female germ cells initiate Polycomb silencing by regulating PRC2-interacting proteins. eLife. 2020; 9: e56922. https://doi.org/10. 7554/eLife.56922 PMID: 32773039

51. Bergman CM, Carlson JW, Celniker SE. Drosophila DNase I footprint database: a systematic genome annotation of transcription factor binding sites in the fruitfly, Drosophila melanogaster. Bioinformatics. 2005; 21: 1747-1749. https://doi.org/10.1093/bioinformatics/bti173 PMID: 15572468

52. Daniel Avery Pollard. Drosophila Sequence Specific Transcription Factor Binding Site Matrices. Available: http://www.danielpollard.com/matrices.html

53. Zhao K, Hart CM, Laemmli UK. Visualization of chromosomal domains with boundary element-associated factor BEAF-32. Cell. 1995; 81: 879-889. https://doi.org/10.1016/0092-8674(95)90008-x PMID: 7781065

54. Roy S, Gilbert MK, Hart CM. Characterization of BEAF Mutations Isolated by Homologous Recombination in Drosophila. Genetics. 2007; 176: 801-813. https://doi.org/10.1534/genetics.106.068056 PMID: 17435231

55. Park J-S, Choi Y-J, Thao DTP, Kim Y-S, Yamaguchi M, Yoo M-A. DREF is involved in the steroidogenesis via regulation of shadow gene. Am J Cancer Res. 2012; 2: 714-725. PMID: 23226617 
56. Tian Y, Smith-Bolton RK. Regulation of growth and cell fate during tissue regeneration by the two SWI/ SNF chromatin-remodeling complexes of Drosophila. Duronio R, editor. Genetics. 2021; 217: 1-16. https://doi.org/10.1093/genetics/iyaa028 PMID: 33683366

57. Liu X, Secombe J. The Histone Demethylase KDM5 Activates Gene Expression by Recognizing Chromatin Context through Its PHD Reader Motif. Cell Reports. 2015; 13: 2219-2231. https://doi.org/10. 1016/j.celrep.2015.11.007 PMID: 26673323

58. Saha N, Liu M, Gajan A, Pile LA. Genome-wide studies reveal novel and distinct biological pathways regulated by SIN3 isoforms. BMC Genomics. 2016; 17: 111. https://doi.org/10.1186/s12864-016-24285 PMID: 26872827

59. Heurteau A, Perrois C, Depierre D, Fosseprez O, Humbert J, Schaak S, et al. Insulator-based loops mediate the spreading of $\mathrm{H} 3 \mathrm{~K} 27 \mathrm{me} 3$ over distant micro-domains repressing euchromatin genes. Genome Biol. 2020; 21: 193. https://doi.org/10.1186/s13059-020-02106-z PMID: 32746892

60. Gurudatta B, Yang J, Van Bortle K, Donlin-Asp P, Corces V. Dynamic changes in the genomic localization of DNA replication-related element binding factor during the cell cycle. Cell Cycle. 2013; 12: 16051615. https://doi.org/10.4161/cc.24742 PMID: 23624840

61. Bernstein BE, Mikkelsen TS, Xie X, Kamal M, Huebert DJ, Cuff J, et al. A bivalent chromatin structure marks key developmental genes in embryonic stem cells. Cell. 2006; 125: 315-326. https://doi.org/10 1016/j.cell.2006.02.041 PMID: 16630819

62. Akmammedov A, Geigges M, Paro R. Bivalency in Drosophila embryos is associated with strong inducibility of Polycomb target genes. Fly (Austin). 2019; 13: 42-50. https://doi.org/10.1080/19336934. 2019.1619438 PMID: 31094269

63. Schertel C, Albarca M, Rockel-Bauer C, Kelley NW, Bischof J, Hens K, et al. A large-scale, in vivo transcription factor screen defines bivalent chromatin as a key property of regulatory factors mediating Drosophila wing development. Genome Res. 2015; 25: 514-523. https://doi.org/10.1101/gr.181305.114 PMID: 25568052

64. De S, Mitra A, Cheng Y, Pfeifer K, Kassis JA. Formation of a Polycomb-Domain in the Absence of Strong Polycomb Response Elements. Bantignies F, editor. PLoS Genet. 2016; 12: e1006200. https:// doi.org/10.1371/journal.pgen.1006200 PMID: 27466807

65. Emberly E, Blattes R, Schuettengruber B, Hennion M, Jiang N, Hart CM, et al. BEAF Regulates CellCycle Genes through the Controlled Deposition of H3K9 Methylation Marks into Its Conserved DualCore Binding Sites. Misteli T, editor. PLoS Biology. 2008; 6: e327. https://doi.org/10.1371/journal.pbio. 0060327 PMID: 19108610

66. Zamurrad S, Hatch HAM, Drelon C, Belalcazar HM, Secombe J. A Drosophila Model of Intellectual Disability Caused by Mutations in the Histone Demethylase KDM5. Cell Rep. 2018; 22: 2359-2369. https:// doi.org/10.1016/j.celrep.2018.02.018 PMID: 29490272

67. Krebs AR, Imanci D, Hoerner L, Gaidatzis D, Burger L, Schübeler D. Genome-wide Single-Molecule Footprinting Reveals High RNA Polymerase II Turnover at Paused Promoters. Molecular Cell. 2017; 67: 411-422.e4. https://doi.org/10.1016/j.molcel.2017.06.027 PMID: 28735898

68. Henikoff S, Henikoff JG, Kaya-Okur HS, Ahmad K. Efficient chromatin accessibility mapping in situ by nucleosome-tethered tagmentation. Genomics; 2020 Apr. https://doi.org/10.7554/eLife.63274 PMID: 33191916

69. Grant CE, Bailey TL, Noble WS. FIMO: scanning for occurrences of a given motif. Bioinformatics. 2011; 27: 1017-1018. https://doi.org/10.1093/bioinformatics/btr064 PMID: 21330290 